\title{
Recipient design aus multimodaler Sicht ${ }^{1}$
}

\begin{abstract}
Der Beitrag ist eine fallbasierte und konzeptionell ausgerichtete Auseinandersetzung mit dem konversationsanalytischen Konzept ,recipient design“. Dieses wird zunächst in seinem monomodal-verbalen Entstehungszusammenhang erläutert und anschließend aus Perspektive der multimodalen Interaktionsanalyse reflektiert. In einem methodologischen Exkurs werden die Verfahren der „visuellen Erstanalyse“ und der „rekurrenten Mehrebenen-Analyse“ vorgestellt und im analytischen Teil umgesetzt. So wird zunächst das visuell wahrnehmbare Verhalten, dann die Äußerungs- und Interaktionsstruktur und abschließend die Prosodie eines lehrerseitigen recipient design analysiert. In einem fallanalytischen Resümee präsentieren wir im Detail das Zusammenspiel der bei der Produktion dieses multimodalen recipient design beteiligten Ausdrucksressourcen. Abschließend diskutieren wir eine Reihe offener Punkte, die bei einer multimodalen Konzeption von recipient design zu beachten sind. Dabei wird deutlich, dass recipient design theoretisch neu gerahmt und systematisch in Bezug auf online-analytische und verstehensdokumentarische Überlegungen reflektiert werden muss.
\end{abstract}

This paper is a case-related, conceptually focused analysis of the conversation analytical concept of recipient design. First, the concept is explained in its monomodal - i.e. verbal - context of origin and subsequently reflected from a multimodal perspective. The techniques of "initial visual analysis" and "recurrent multilevel analysis" are then introduced in a methodological excursus and applied in the analysis section. This way, the visually perceivable behavior is analyzed first, followed by the reconstruction of both utterance and interaction structure. Finally, the prosody of a teacher's utterances is analyzed. The summary presents in detail the interplay of the resources which produce the multimodal recipient design. In conclusion, a number of open issues to be considered in the case of a multimodal conception of recipient design are discussed. This highlights the fact that recipient design requires a new theoretical framework, as well as a systematic reflection with regard to online-analytical considerations and the documentation of understanding.

\section{Einleitung}

Die videobasierte Analyse von Unterrichtsinteraktionen zwischen Lehrer/innen und Schüler/innen (Schmitt 2009, 2011, 2012d) hat uns zur Frage geführt, in welcher Weise der jeweilige Interaktionspartner sein Gegenüber durch sein eigenes Verhalten ,repräsentiert“. Es geht also im weitesten Sinne um Fragen wie:

- Durch welche Verhaltensweisen bzw. durch welche Aspekte seines Verhaltens zeigt der Lehrer einem Schüler, als was/wen er ihn (in der Situation) sieht und welche Beziehung er (in der Situation und vielleicht auch darüber hinaus) zu ihm hat?

- Worauf reagiert diese Form der wahrnehmbaren Repräsentanz? Bringt der Lehrer sie mit oder reagiert sie auf die spezifische Präsenz des Schülers in der aktuellen Situation?

- Reagiert der Schüler seinerseits auf die Art und Weise, wie der Lehrer ihm wahrnehmbar macht, als wen er ihn sieht?

1 Wir bedanken uns bei den Teilnehmer/innen des Forschungskolloquiums im IDS (Serap Öndüc, Nikolina Pustički, Jenny Winterscheid, Reinhard Fiehler, Ulrich Reitemeier und Axel Schmidt) sowie Arnulf Deppermann für gemeinsame Analysen, konstruktive Kritik und Beiträge zum hier vorliegenden Text. 
Diesen Fragen wollen wir auf der Grundlage einer videobasierten Fallanalyse mit einem multimodalen Erkenntnisinteresse und auf der Grundlage einer konstitutionsanalytischen Methodologie nachgehen. In konzeptioneller Hinsicht orientieren wir uns dabei an der konversationsanalytischen Vorstellung von recipient design. Der Aufsatz versteht sich insgesamt als Beitrag zur Weiterentwicklung des Ansatzes einer multimodalen Interaktionsanalyse (MIA) sowohl in methodischer als auch konzeptioneller Hinsicht.

Zunächst werden wir relevante Aspekte der multimodalen Interaktionsanalyse skizzieren, die für den hiesigen Erkenntniszusammenhang wichtig sind (Abschnitt 2). Dann präsentieren wir das Konzept ,recipient design“ in seinem konversationsanalytischen Verständnis, um daran die eigene Reflexion anzuschließen, die sich aus der Notwendigkeit des multimodalen Zuschnitts von recipient design ergibt (Abschnitt 3).

Im Anschluss werden wir einen methodologischen Exkurs unternehmen, der sich aufgrund der audiovisuellen Qualität unseres Falles hinsichtlich der konstitutionsanalytischen Rekonstruktion eines lehrerseitigen recipient design unter den organisations- und interaktionsstrukturellen Bedingungen seiner Produktion ergibt (Abschnitt 4).

Bei der anschließenden Fallanalyse kombinieren und demonstrieren wir zwei (bislang eher beschriebene als systematisch angewandte) Analyseverfahren: die „visuelle Erstanalyse“ und die „systematische Mehrebenen-Analyse“. Wir analysieren dabei zunächst alle relevanten visuell wahrnehmbaren Ausdrucksressourcen der uns interessierenden Beteiligten. Erst im Anschluss wenden wir uns der Verbalität in Form einer äußerungs- und interaktionsstrukturellen Analyse zu. Dabei werden wir auch die spezifische interaktive Leistung der Prosodie als Teil des multimodalen recipient design berücksichtigen (Abschnitt 5).

Nach einem fallspezifischen Resümee, in dem wir den systematischen Zusammenhang aller für das recipient design relevanten Ausdrucksressourcen im Detail zeigen (Abschnitt 6), weisen wir offene Punkte und Probleme des Konzeptes aus multimodaler Sicht aus (Abschnitt 7).

\section{Multimodale Interaktionsanalyse (MIA)}

Da die Entstehung und bisherige Entwicklung einer multimodalen Konzeption von Interaktion bereits sehr gut dokumentiert ist ${ }^{2}$, können wir uns damit begnügen, auf diejenigen Aspekte zu verweisen, die für unser spezifisches Erkenntnisinteresse zentral sind.

Die multimodale Interaktionsanalyse ist ein Zugang, der sich aus unterschiedlichen Quellen speist. Für unser spezifisches Interesse sollen hier - und auch das nur in sehr aspektualisierter Form - zwei Verweise genügen: auf die context analysis und die Konversationsanalyse.

Die context analysis als Bezugspunkt ist vor allem hinsichtlich des Postulats der theoretischen Egalität aller Ausdrucksressourcen wichtig. Der Ansatz betont ,the importance of an integrated approach to the study of interaction“ und ,,refuses to assume that any particular modality of communication is more salient than another" (Kendon 1990b, S. 16). ${ }^{3}$

Einen systematischen Überblick über die deutschsprachige Entwicklung des multimodalen Ansatzes liefern die thematisch aufeinander bezogenen Bände: Schmitt (Hg.) (2007), Mondada/Schmitt (Hg.) (2010), Hausendorf/Mondada/Schmitt (Hg.) (2012) und Schmitt (2013).

3 Zur context analysis siehe weiterhin Scheflen (1964, 1972), Heilman (1979) sowie Birdwhistell (1970, 1984). 
Die Vorstellung der context analysis, dass das verbale Primat bei der Untersuchung von Kommunikation zu Gunsten eines integrierten Ansatzes aufgegeben werden muss, der alle Ausdrucksressourcen bei der Analyse und theoretischen Konzeption von Kommunikation berücksichtigt, ist für das Selbstverständnis der multimodalen Interaktionsanalyse zentral. Zu weiteren Übereinstimmungen zählt neben diesem methodologisch implikationsreichen Egalitätspostulat auch die konzeptionelle Relevanz, die ,Simultaneität‘ neben ,Sequenzialität' als zentraler generativer Mechanismus bei der Konstitution interaktiver Strukturen besitzt. ${ }^{4}$ Terminologisch ist das Konzept ,multimodale Kommunikation ' bereits bei Scheflen (1972, S. 230) vorgeprägt, der von Körperpositur als einer ,modality of communication" spricht.

Der konversationsanalytische Ansatz spielt für die Entwicklung der multimodalen Perspektive deswegen eine zentrale Rolle, weil ein Großteil der Linguist/innen, die an der Analyse, Konzeptualisierung und methodologischen Reflexion der multimodalen Qualität von Interaktion interessiert sind, einen konversationsanalytischen Hintergrund haben und auf der Basis der konstitutionsanalytischen Methodologie und grundlagentheoretischen Ausrichtung dieses Ansatzes arbeiten.

Obwohl die multimodale Interaktionsanalyse ein stabiles Standbein in der Konversationsanalyse hat, ist sie doch mehr als ein Zusatz oder eine einfache Weiterentwicklung. Auch wenn inzwischen im konversationsanalytischen Forschungskontext die empirische Grundlage nicht mehr nur aus Tonbandaufzeichnungen besteht, sondern aus Videodokumentationen, wird das Erkenntnisinteresse doch weitgehend vom Primat der Verbalität als zentrale Ressource der Interaktionskonstitution bestimmt.

Im Moment wird die aktuelle Entwicklung der konstitutionsanalytischen Untersuchung von Kommunikation durch ein Kontinuum koexistierender Zugänge bestimmt, die sich bezüglich der theoretisch-methodisch-konzeptionellen Bedeutung von Verbalität unterscheiden.

Die klassische Konversationsanalyse: Untersuchungen auf der Grundlage dieser Konzeption arbeiten nach wie vor mit Audioaufzeichnungen und gestalten ihre Gegenstandskonstitution so, dass Phänomene und Strukturen im Mittelpunkt des Erkenntnisinteresses stehen, die auf der Grundlage einer monomodalen und als autonom behandelten Ausdrucksressource, der Verbalität, untersucht werden.

Die multimodale Konversationsanalyse: Arbeiten mit diesem Schwerpunkt integrieren visuelle Ausdrucksressourcen bei der multimodalen Erweiterung verbal definierter Konzepte. Sie arbeiten daher auf der Grundlage audio-visueller Interaktionsdokumente. Das Erkenntnisinteresse wird auch hier weitgehend noch von Strukturen verbaler Kommunikation bestimmt. Es geht dabei beispielsweise um Fragen, wie verbale Mechanismen der Interaktionskonstitution wie etwa die Turn-Taking-Organisation durch Blickverhalten, Gestikulation, Mimik etc. mitbestimmt werden (zu Turn-Taking beispielsweise Mondada 2007; Schmitt 2005, zu Pausen Schmitt 2004).

Die multimodale Interaktionsanalyse: Dieser Ansatz benutzt ausschließlich Videoaufzeichnungen, hat aber im Unterschied zur multimodalen Konversationsanalyse per se kein primäres Interesse an der Rekonstruktion verbaler Strukturen. Das Erkenntnisinteresse

4 Kendon (1990b, S. 49) verweist darauf, dass es große Gemeinsamkeiten zwischen context analysis und dem talk-in-interaction-Ansatz gibt. 
bezieht sich vielmehr auf die Rekonstruktion interaktiver Ordnungsstrukturen. Für den Ansatz ist aufgrund seiner interaktionstheoretischen Grundlage die Selbstbezeichnung „multimodale Interaktionsanalyse“ in ihrer Kontrastivität zum einen wissenschaftsgeschichtlich motiviert, zum anderen ein Pleonasmus: Interaktion ist in ihrem situativen Vollzug immer multimodal!

Zwischen diesen drei Formen konstitutionsanalytischer Rekonstruktion, die ihre gemeinsame methodologische Grundlage in der ethnomethodologischen Konstitutionsvorstellung einer Vollzugsrealität besitzen, besteht einerseits eine große Durchlässigkeit, andererseits gibt es auch deutliche Unterschiede. Letztere beziehen sich vor allem auf die theoretisch motivierte Art der Gegenstandskonstitution und auf die Rolle, welche die bei der Analyse verbaler Interaktion entwickelten Konzepte spielen.

Im Unterschied zu den beiden ausschließlich bzw. stärker verbal orientierten Zugängen ist für die multimodale Interaktionsanalyse charakteristisch, dass alle Formen interaktiver Praxis, ungeachtet der bei ihrer Konstitution eingesetzten Ausdrucksressourcen, gleichwertige Untersuchungsgegenstände sind. Das führt im Einzelfall (nicht grundsätzlich!) zur methodisch motivierten Fokussierung von Visualität als bislang weitgehend vernachlässigter Konstituente der Interaktion (siehe etwa Schmitt 2012a, b und Schmitt, erscheint).

Da Überlegungen zu einer multimodalen Konzeption von Interaktion sowohl wissenschaftshistorisch als auch hinsichtlich ihrer methodologischen und theoretischen Grundlagen enge Beziehungen zur Konversationsanalyse besitzen, rücken quasi naturwüchsig etablierte konversationsanalytische Konzepte in den Fokus. Dies umso mehr, als Verbalität eine zentrale und am besten untersuchte Ausdrucksressource darstellt und die Konversationsanalyse seit den 1960er Jahren erfolgreich und systematisch grundlegende Mechanismen der verbalen Interaktion untersucht und konzeptionell aufgearbeitet hat.

Aus multimodaler Sicht besitzen die konversationsanalytischen Konzepte zum einen eine starke und produktive Attraktivität, zum anderen sind sie aufgrund ihrer monomodalen Qualität nicht unproblematisch. Monomodale Konzepte konterkarieren das theoretische Postulat der Egalität aller Ausdrucksressourcen und die damit zusammenhängenden analysemethodischen Folgen. Immer dann, wenn sich die Orientierung an monomodal-verbalen Konzepten als Folge multimodaler Analysepraxis ergibt, ist man (zum jetzigen Zeitpunkt und wahrscheinlich noch für eine ganze Weile) gezwungen, diese Konzepte auf ihre Tauglichkeit für den multimodalen Erkenntniszusammenhang zu prüfen.

Wir selbst sind bei unseren Analysen auf diesem Wege auf das Konzept „recipient design“ gestoßen. Dieses Konzept wollen wir nachfolgend aus Sicht der multimodalen Interaktionsanalyse fallbasiert reflektieren und auf seine Multimodalitätstauglichkeit befragen.

\section{Die Repräsentation des Anderen im eigenen interaktiven Verhalten}

Bevor wir in die Analyse einsteigen, wollen wir unser Verständnis von recipient design darlegen und damit den konzeptionellen Rahmen aufspannen, in dem sich unsere analytischen Bemühungen erkenntnisbezogen bewegen.

Interaktion ist nicht nur strukturell betrachtet ein dialogisches Unternehmen, bei dem wir es mit einem Gegenüber zu tun haben, auf dessen Präsenz wir in Form initiativer und reaktiver Züge reagieren. Wir stellen nicht nur Fragen an unser Gegenüber und antworten diesem, sondern reagieren auf unser Gegenüber in grundlegender und letztlich nicht hin- 
tergehbarer Weise in unserem gesamten Interaktionsverhalten. In der Art und Weise, wie wir in seiner Gegenwart grundlegende interaktive Anforderungen bearbeiten, ob und wie wir auf gemeinsames Wissen zurückgreifen oder dieses fraglos unterstellen, als wen wir unser Gegenüber sozial „,sehen“ und einschätzen, ob und wie wir uns selbst mit unserem Gegenüber in Beziehung setzen etc., drückt sich - ob wir wollen oder nicht - in unterschiedlichen Aspekten unseres interaktiven Gesamtverhaltens aus. Zu interagieren bedeutet in diesem Sinne, unser Gegenüber (und uns selbst) kontinuierlich in unserem Verhalten zu repräsentieren. Damit wird unser Verhalten für unser Gegenüber zu einer ergiebigen Informationsquelle, aus der er/sie das Bild, das wir von ihm/ihr haben, herauslesen und selbst wieder in seine/ihre eigene interaktive Präsenz integrieren kann.

Dieser kontinuierliche Bezug auf und die permanente Repräsentanz unseres Gegenübers wird im Hinblick auf seine interaktive Evidenz und seine Multiaspektualität von unterschiedlichen Ansätzen vor allem als verbales Phänomen gesehen und konzeptualisiert.

\subsection{Ein kurzer Konzeptüberblick}

Recipient design ist nur eines von mehreren Konzepten, mit denen der Zuschnitt von Äußerungen und die Strukturierung von Turns für Rezipienten bezeichnet werden. Neben der Konversationsanalyse spielt dieser Aspekt unter anderem für folgende Ansätze eine Rolle: ${ }^{5}$

- Die soziolinguistische Höflichkeitsforschung untersucht die Abhängigkeit von Adressierung und Höflichkeitsformen in Abhängigkeit von Bekanntheit, Status und Sympathie zwischen Gesprächsbeteiligten (vgl. Brown/Levinson 1987).

- Die sozialpsychologische Forschung zur Akkomodation (Giles/Coupland 1991) und das sozialstilistische Konzept des audience design (Bell 2001) betrachten die sprachliche Anpassung an das Register bzw. den Code des Adressaten (s.a. Coupland 2007, S. 54-81).

- In der Medienforschung wurde untersucht, wie mit medialen Kommunikaten unterschiedliche Sprechakte für verschiedene Teilpublika vollzogen werden (Kühn 1995; Holly 1990). Ähnlich gelagerte Fragen nach dem Zusammenhang zwischen verschiedenen Bedeutungen der gleichen Äußerung mit unterschiedlichen Beteiligungsrollen von Rezipienten in Mehrparteieninteraktionen fasst Clark (1992) mit seinem (nicht mit Bell zu verwechselnden) Konzept audience design.

- Die allgemeine Relevanz der Zuschreibung von geteiltem Wissen an den Gesprächspartner (,shared knowledge“ bzw. „,common ground“) für die Gestaltung von Interaktionsbeiträgen ist ein zentrales Thema der kognitionswissenschaftlich ausgerichteten Untersuchung von Interaktionen (z.B. Clark 1996a) und ihrer sprachlichen Gestaltung (Clark 1996 b; Fetzer/Fischer 2007 und auch Gumperz 2002).

In den unterschiedlichen Konzepten der Adressatenorientierung spielen verschiedene Aspekte eine zentrale Rolle: Das sind zum einen Eigenschaften der Beteiligten wie sozialer Status, Wissen, interaktiver Beteiligungsstatus etc.; zum anderen werden sehr unterschiedliche Verfahren bzw. Aspekte des verbalen Adressatenzuschnitts erfasst wie Formulierungswahl und Codeselektion, grammatikalische Markierungen, sequenzielle Organisation, Referenzformen, Sprechakttypen, Anspielungen etc.

Den nachfolgenden Überblick übernehmen wir von Schmitt/Deppermann (2010, S. 81). 


\subsection{Die konversationsanalytische Konzeption „recipient design“}

„Recipient design“ als originär konversationsanalytisches Konzept wurde von Sacks (1992) in den frühen 1970er Jahren entwickelt (vgl. Malone 1997, S. 100-119). In der Konversationsanalyse wurde frühzeitig erkannt, dass ein systematischer Zusammenhang existiert zwischen der Art und Weise, in der ein Sprecher Äußerungen für einen bestimmten Hörer/Rezipienten formuliert, und den Annahmen, die der Sprecher damit über den Rezipienten zum Ausdruck bringt.

Einerseits sind Interaktionsbeiträge nicht-hintergehbar recipient designed, d.h., immer an spezifische Rezipienten und das aus deren interaktiver Präsenz erschließbare Wissen um grundlegende Relevanzen der aktuellen Situation sowie an deren interaktionsgeschichtlichen und normalformspezifischen Grundlagen angepasst. Andererseits werden diese Beteiligten dadurch in der Interaktion als bestimmte Rezipienten mit spezifischen Eigenschaften positioniert. ${ }^{6}$

Sacks/Schegloff/Jefferson (1974, S. 727) definieren das Konzept in seiner Grundstruktur wie folgt:

By ,recipient design' we refer to a multitude of respects in which the talk by a party in a conversation is constructed or designed in ways which display an orientation and sensitivity to the particular other(s) who are co-participants.

Als zentrale Konstituenten des Konzeptes werden Vielfältigkeit der empirischen Manifestation des Konzeptes (,,multitude of respects“), die Ausschließlichkeit von Verbalität (,talk in a conversation"), der klare Sprecherbezug (,talk by a party") sowie die Orientierung an Ko-Beteiligten und Sensitivität für sie deutlich.

Hinsichtlich der empirischen Manifestation von Aspekten, mit denen recipient design ausgedrückt bzw. formuliert werden kann, nennen die Autoren (ebd.) die folgenden:

In our work, we have found recipient design to operate with regard to word selection, topic selection, admissibility and ordering of sequences, options and obligations for starting and terminating conversations etc.

Wir interessieren uns für recipient design nicht als situationstranszendente Konstante des Verhaltens von Interaktionsbeteiligten (dafür gibt es Konzepte wie etwa „Präsenzfigur“; Schmitt 1992a [2008], 1992b). Wir beschäftigen uns fallanalytisch und konzeptuell vielmehr mit einer Form, die in spezifischen interaktionsstrukturellen Konstellationen situationssensitiv, lokal-spezifisch und in Reaktion auf vorangegangenes Interaktionsgeschehen realisiert wird. Solche Realisierungen von recipient design sind dadurch charakterisiert, dass sie in ihrer lokalen Relevanz und als motivierte Ressource der Interaktionskonstitution eingesetzt und wahrnehmbar gemacht werden.

Wir knüpfen für unsere Analyse eng an das konversationsanalytische Konzept „recipient design“ an. Die Gründe hierfür liegen in der konzeptionellen Relevanz folgender Aspekte, die - wenn auch nicht in gleicher Terminologie - auch für die konversationsanalytische Vorstellung charakteristisch sind:

6 Eine solche Positionierung als dominanter Aspekt eines recipient design wird ausführlich in Schmitt/ Deppermann (2009) analysiert. Zum Konzept der (Fremd-)Positionierung in Narrationen siehe LuciusHoene/Deppermann (2002, Kap. 8.2; 2004) sowie Deppermann (erscheint). 
a) Relevanz von Wissensbeständen: Damit ist die Abhängigkeit des Interaktionshandelns von Annahmen über den Partner gemeint, insbesondere über seine für den aktuellen Interaktionszusammenhang relevanten Wissensbestände ${ }^{7}$,

b) strukturelle Reaktivität: Damit treten beobachtbare Verfahren der Entwicklung und Anpassung der Gestaltung des recipient design an das multimodale Verhalten spezifischer, identifizierbarer Beteiligter in einer fokussierten Interaktion ins Blickfeld. Diese Gestaltungsmittel und Verfahren werden rekonstruiert in ihrem empirisch evidenten Bezug auf nahkontextuell oder zurückliegendes Partnerverhalten,

c) sequenzielle Intersubjektivierung: Hiermit ist die grundsätzliche, empirisch rekonstruierbare Drei-Phasen-Struktur gemeint, in der sich die interaktive und lokale Ausrichtung, Aushandlung und Angemessenheit/Unangemessenheit des recipient design als gemeinschaftlich hergestellte Sequenz von Angebot - Reaktion - Ratifikation vollzieht.

Diese Aspekte sind für den von uns untersuchten Fall grundlegend. Dabei geht es darum, wie in Reaktion auf eine verbal abstinente (Heidtmann/Föh 2007) Selbstpräsentation eines Schülers, der etwas verspätet in den Unterricht kommt, dieser vom Lehrer in und für die Öffentlichkeit der Klasse quasi aus dem Stand als Rezipient mit spezifischen Eigenschaften konstituiert wird. Es ist zu sehen, wie der Schüler dieses recipient design des Lehrers als Ratifikation seines verhaltensspezifischen Angebots interpretiert und auf der Grundlage dieser Ratifikation weiter agiert und damit das recipient design des Lehrers intersubjektiv als kollektive, konvergierende Orientierung für die weitere Interaktion behandelt.

Im Fokus unseres Erkenntnisinteresses steht also die lokale Herausbildung und kollektive Herstellung eines spezifischen Rezipienten. Unsere Fallspezifik verweist jedoch gleichzeitig situationstranszendierend auf die Relevanz interaktiver Vorgängigkeit. Sie ist ein wichtiger Bezugspunkt, der von den Beteiligten selbst ausgewiesen wird, indem sie den situativen Vollzug in seiner dokumentarischen Qualität kenntlich machen: für sich als unmittelbar Beteiligte und verbal Aktive, sowie für die Klassenöffentlichkeit als relevante Ko-Beteiligte. Die dreischrittige Konstitution des recipient design (Angebot - Reaktion - Ratifikation) ist sowohl eine stabile intersubjektive Orientierung als auch der Relevanzrahmen, in dem die Beteiligten ihr Verhalten organisieren.

\section{Visuelle Erst- und rekurrente Mehrebenen-Analyse}

Die an der Strenge der konversationsanalytischen Methodologie orientierte konstitutionsanalytische Rekonstruktion multimodaler Interaktionsstrukturen verlangt die Entwicklung spezifischer, auf die Eigenschaften des Untersuchungsgegenstandes bezogener Analyseverfahren. Das komplexe Zusammenspiel unterschiedlicher Ausdrucksressourcen erfordert Verfahren, die neben der Sequenzialität auch dem Prinzip der Gleichzeitigkeit in adäquater Weise Rechnung tragen. Zudem kann man bei der Rekonstruktion multimodaler Ausdrucksweisen nicht alle relevanten Aspekte gleichzeitig erfassen, sondern muss sich in wiederholten Durchgängen durch den zu analysierenden Videoausschnitt auf jeweils einzelne Ausdrucksaspekte konzentrieren (Schmitt 2007a, b).

\footnotetext{
Konversationsanalytische Schwerpunkte der Untersuchung von recipient design sind Formulierung von Raum- und Personenreferenzen in Abhängigkeit vom angenommenen bzw. aus dem Gesprächsverlauf zu erschließenden Partnerwissen und deren Korrektur (Sacks/Schegloff 1979; Schegloff 1972, 2007; Heritage 2007).
} 
Dabei scheint es zunächst naheliegend, mit der Analyse des verbalen Geschehens zu beginnen und die Rekonstruktion der anderen Ausdrucksressourcen darauf aufzubauen. Ein wesentliches Argument für ein solches analysefaktisches Primat der Verbalität ist die methodische Sicherheit, über die wir aufgrund der Erkenntnisse der konversationsanalytischen Auseinandersetzung mit Gesprächen verfügen. Zudem scheint ein verbaler Einstieg naheliegend, weil in vielen Fällen die voranalytische Selektion relevanter Videoausschnitte auf der Grundlage des Gesprochenen erfolgt.

Die analysefaktische Priorisierung des Verbalen ist jedoch nicht nur problematisch, weil es die theoretisch postulierte Egalität aller Modalitätsebenen, die ein wesentliches Fundament der multimodalen Konzeption von Interaktion darstellt, bei der Analyse unterläuft. Eine Priorisierung der verbalen Ausdrucksressource ist vielmehr in vielen Fällen sowohl analysefaktisch als auch in gegenstandskonstituierender Hinsicht erkenntnishemmend.

$\mathrm{Ob}$ und wie weit der rekurrente, jeweils einzelne Ausdrucksressourcen fokussierende analytische Durchgang durch dasselbe Videosegment fallunabhängig methodisiert werden kann, lässt sich nur durch systematische fallanalytische Explorationen beantworten. Sicher ist jedoch, dass es nötig sein wird, dabei die eigene „De-facto-Methodologie“ systematisch zu rekonstruieren, der man bei der Analyse relevanter Ausdrucksressourcen folgt. ${ }^{8}$

Wir haben zum Zwecke der Fokussierung der körperlichen Ausdrucksformen die vokale Ebene zunächst motiviert ausgeschlossen und uns initial nur mit dem visuell wahrnehmbaren Verhalten der Beteiligten beschäftigt. Eine solche methodisch motivierte Entscheidung führt zur visuellen Erstanalyse als einer Variante der Modalitätsfokussierung. Der Begriff „visuelle Erstanalyse“ verweist auf die spezifische, durch den Forschungsgang definierte Position und Funktionalität dieses Verfahrens: Nur wenn die Analyse des visuellen Interaktionsverhaltens noch nicht durch das Wissen um das verbale Geschehen beeinflusst ist, ist es sowohl hinsichtlich der Identifikation neuer, für die Interaktionskonstitution relevanter Aspekte als auch in deskriptiv-kategorialer Hinsicht produktiv.

Wird ein Videoausschnitt auf der Basis der konstitutionsanalytischen Methodologie und unter Ausschluss der verbalen Anteile segmentiert und in seiner sichtbaren Struktur beschrieben, führt das zwangsläufig zur Entwicklung neuer Beschreibungskategorien. Die visuelle Erstanalyse ist in dieser Hinsicht ausgesprochen implikationsreich und erkenntnisgenerierend. Bei der detaillierten Deskription visueller Verhaltensaspekte bildet sich gerade durch das Fehlen von auf Verbalität bezogener kategorialer Vorverständigkeit quasi naturwüchsig eine strukturreflexive deskriptive Beschreibungssprache aus. Diese muss jedoch vom Analytiker häufig in ihrer strukturreflexiven Qualität erst einmal erkannt werden, um sie systematisch als Erkenntnismöglichkeit nutzen zu können. Dann wird deutlich, dass dieser deskriptive Beschreibungsmodus geeignet ist, spezifische, rein visuell wahrnehmbare Relevanzstrukturen zu identifizieren, und dazu beiträgt, visuell wahrnehmbare Aspekte der Interaktionskonstitution in ihrer spezifischen pragmatischen Wertigkeit in den Blick zu nehmen.

Doch welchen pragmatischen Gehalt besitzen die mittels des deskriptiven Modus identifizierten visuellen Verhaltensaspekte? Man kann sich die Implikationen des Verfahrens an folgendem Beispiel verdeutlichen: Bei der visuellen Erstanalyse eines Videoausschnitts vom Filmset (Schmitt/Deppermann 2007) konnte man sehen, wie eine Regisseurin ihre

8 Die konversationsanalytische „De-facto-Methodologie“ und ihre „weißen Flecken“ im faktischen Forschungshandeln sind detailliert ausgeführt in Schmitt (2001). 
Kamerafrau durch eine Umarmung und eine gleichzeitig realisierte Bewegung um die eigene Achse „umdreht“. Nachdem auf der verbalen Ebene nachfolgend eine Auseinandersetzung zwischen den beiden rekonstruiert werden konnte, wurde deutlich, dass das Umdrehen auch im Sinne eines gesprächsrhetorischen Zuges verstanden werden kann ${ }^{9}$. Es hat dann die pragmatische Implikation von „Jemanden von seiner ursprünglichen Position abbringen und ihn in die eigene Richtung orientieren". Dies ist ein instruktives Beispiel dafür, dass relevante pragmatische Orientierungen der Beteiligten nicht nur verbalisiert werden, sondern auch als Verkörperung zum Ausdruck kommen. Es lohnt sich also, sich für die Frage zu sensibilisieren, ob sich vergleichbare, visuell gestützte Beobachtungen wie „Regisseurin dreht ihre Kamerafrau um“ in systematischer Weise als Erkenntnisinstrument nutzen lassen und wie weit solche Deskriptionen dazu beitragen, relevante Aspekte der interaktiven Ordnungsstruktur zu fokussieren.

Es gibt unterschiedliche Argumente für die Produktivität und Notwendigkeit, das Verfahren der visuellen Erstanalyse einzusetzen.

- Es kann sein, dass die Spezifik der Daten selbst dieses Verfahren „einfordert“ (siehe Schmitt 2004),

- das Verfahren ist die analysefaktische Umsetzung des aus der context analysis stammenden theoretischen Postulats der Egalität aller Ausdrucksressourcen,

- es operiert auf der Grundlage eines interaktionstheoretisch und wissenschaftshistorisch motivierten „Misstrauens gegenüber der fraglosen Macht des Verbalen“ in gegenstandskonstitutiver, konzeptioneller und analysemethodischer Hinsicht,

- es sensibilisiert für autonome Konstitutionsleistungen des visuell wahrnehmbaren Interaktionsverhaltens,

- es trägt bei zur Entwicklung eines visuell basierten „deskriptiven Modus“ in Form einer strukturhaltigen und relevanz-transparenten Begrifflichkeit und die Explikation der darin enthaltenen pragmatischen Implikationen und Konzepte, die aufgrund ihrer Deskriptivität ,näher“ an den Beteiligtenorientierungen „,dran“ sind: Beispiele hierfür sind: ,jemanden umdrehen“ (Schmitt/Deppermann 2007), ,jemanden auf den Boden zurückholen“(Pustički/Schmitt i.V.) und „Seitenstechen inszenieren“ (im analysierten Fall),

- es eröffnet Zugang zur interpretativen Kompetenz, die es uns erlaubt, aus interaktiven Vorgängen, die wir nur sehen (nicht hören), relativ präzise „Seh-Arten“ zu entwickeln,

- sie ermöglicht die Entwicklung von Gegenständen und Fragestellungen, die gegenüber Verbalität als Aspekt der Interaktionskonstitution autonom sind (beispielsweise „Gehen als situierte Praktik“ (Schmitt 2012a), „Be-Greifen“ relevanter Aspekte der sozial-räumlichen Umgebung (Schmitt, erscheint),

- es ermöglicht die empirische Substantiierung relevanter Konzepte wie „online-Analyse" und

9 Zu den theoretischen Implikationen der Gesprächsrhetorik siehe Kallmeyer (1996) sowie als Exemplifikation des Ansatzes (Kallmeyer/Schmitt 1996). 
- es erweitert den Bereich verstehensdokumentarischer Aktivitäten (Deppermann 2008; Deppermann/Schmitt 2009; Schmitt 2010) und erlaubt dadurch die Permanenz der Intersubjektivitätskonstitution in seiner ganzen empirischen Vielfalt deutlich zu machen.

Wir haben unsere Analyse nach den Verfahren der visuellen Erst- und der rekurrenten Mehrebenen-Analyse durchgeführt und detailliert ausgearbeitet. Leider können wir dieses Vorgehen hier aus Platzgründen nicht demonstrieren. Unsere Ergebnispräsentation beschränkt sich notgedrungen auf eine Kombination aus genereller Ergebnisbeschreibung und punktueller Detaillierung, wenn es um Verhaltensaspekte geht, die besondere prototypische Qualität für unser Erkenntnisinteresse besitzen.

\section{Die Fallanalyse}

Nach diesen methodologischen Bemerkungen wollen wir uns nun konstitutionsanalytisch unseren Daten zuwenden. Wir rekonstruieren dazu zunächst den relevanten Kontext, in dem das multimodale recipient design des Lehrers realisiert wird. Es ist für unser Erkenntnisinteresse von Bedeutung, ob das analysierte Verhalten des Lehrers dem Schüler gegenüber demjenigen entspricht, das er auch anderen Schülern gegenüber realisiert - es sich somit um so etwas wie eine lehrerspezifische Normalform (Cicourel 1975) handelt - oder ob sich darin etwas Spezifisches und Markiertes ausdrückt. Im ersten Fall würde der Lehrer den eintretenden Schüler eher kategorial ,als Schüler“ positionieren; träfe Letzteres zu, wäre damit eher eine Positionierung als individualisierter Unterrichtsteilnehmer verbunden. Grundlegend orientieren wir uns dabei an der von Goodwin (2003) formulierten, letztlich aber auf die context analysis zurückgehende Perspektive, dass die soziale Bedeutung eines interaktiven Verhaltens (hier recipient design) in vielen Fällen nicht ohne die Analyse des Kontextes rekonstruiert werden kann, in dem das Verhalten realisiert wird.

Der Schüler betritt zu einem Zeitpunkt den Raum, zu dem sich eine Schülerin beim Lehrer und dessen Pult aufhält. Die Schülerin geht in der Vorphase des Unterrichts nach vorne, um - wie zwei Mitschülerinnen unmittelbar vor ihr - ihre Aufnahmezustimmung abzugeben. Diese Interaktion Schülerin-Lehrer stellt die kontrastive Bezugsfolie für die nachfolgende Interaktion zwischen dem Lehrer und dem Schüler dar. Dabei sind die zwischen beiden Interaktionen existierenden handlungstypologischen und organisationsstrukturellen Unterschiede für die Analyse des recipient design wichtig, da sie Fragen nach der aktivitätsspezifischen Sensibilität von recipient design eröffnen: Die Schülerin interagiert mit dem Lehrer als etablierte Teilnehmerin des bereits in Vorbereitung befindlichen Unterrichts. Der Schüler hingegen kommt „etwas zu spät“ in den Klassenraum und „stört“ dadurch den etablierten Unterrichtszusammenhang und die institutionelle Ordnung.

\subsection{Visuelle Erstanalyse}

\subsubsection{Schülerin-Lehrer-Dyade}

Beim Gang nach vorne ist der Blick der Schülerin kontinuierlich auf den Unterlagenstapel auf dem Lehrerpult gerichtet, wo schon mehrere Aufnahmezustimmungen liegen (Bild 1, 2). 
(Hausendorf 2003) realisiert wird, reagieren neben dem Lehrer auch einige Schüler as Auftauchen des Klassenkameraden (Bild 8).

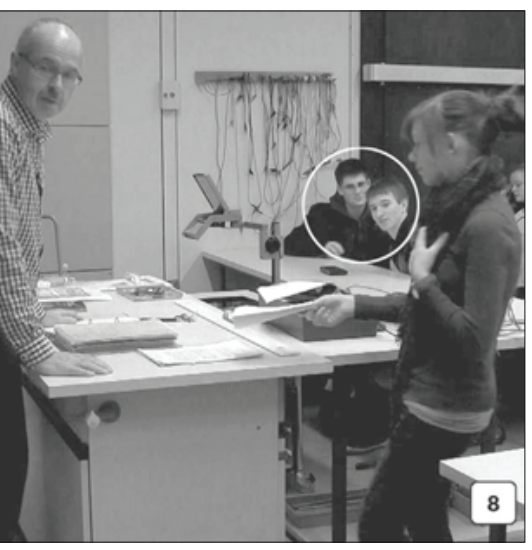

end sich der Schüler in den Raum hinein und auf den Lehrer zubewegt, schaut er n kontinuierlich an. Sein Gesichtsausdruck hat sich inzwischen zu einem deutlichen ln verändert und sein Mund ist weiter geöffnet (Bild 9a, b). In unmittelbarer Reakuf das Einsetzen des Schülerlächelns kommt es zu einer Bewegung auf Seiten des rrs.
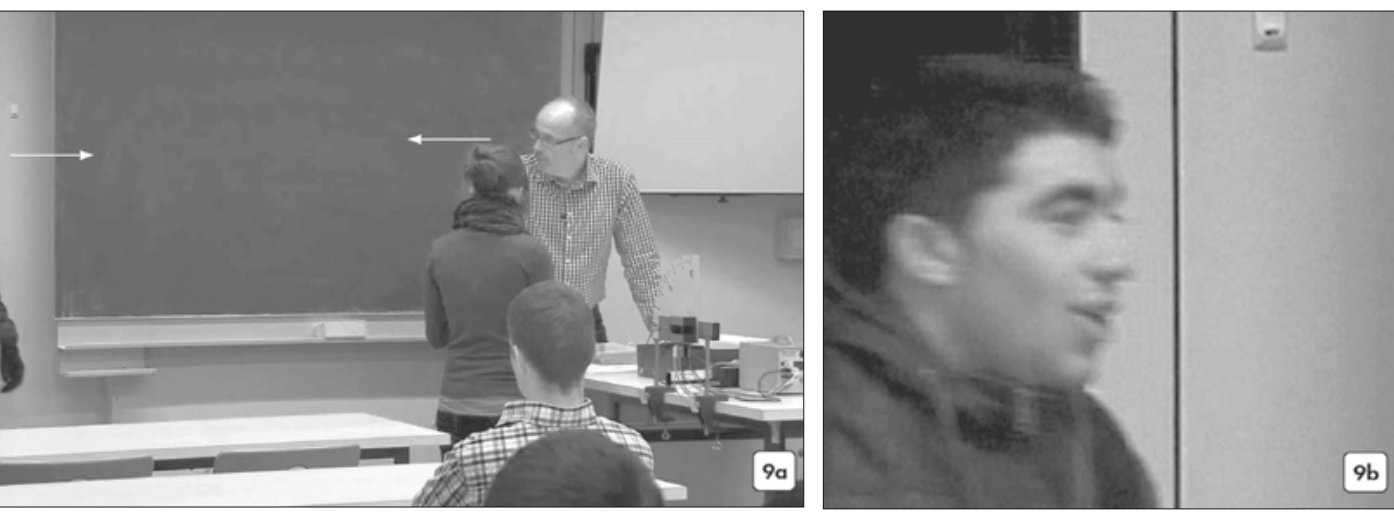

ehrer reagiert expressiv auf den Schüler. Das markante Wegdrehen des Kopfes, desimische Begleitaspekte und vor allem der konkrete Zeitpunkt können als schnelle kation der angebotenen Interaktionsmodalität betrachtet werden. Er wendet unmit- nach Beginn des Lächelns seinen Kopf in einer deutlichen Drehung nach links vom er weg. Sein Kopf beschreibt eine kreisförmige, zunächst leicht nach oben und dann unten verlaufende Bewegung. Mit Beginn dieser Kreisbewegung runzelt der Lehrer ch die Stirn, schließt die Augen und bewegt seine Lippen (Bild 10, 11, 12, 13, 14). fnet seine Augen erst wieder als er nach unten auf sein Pult schaut (Bild 15). 

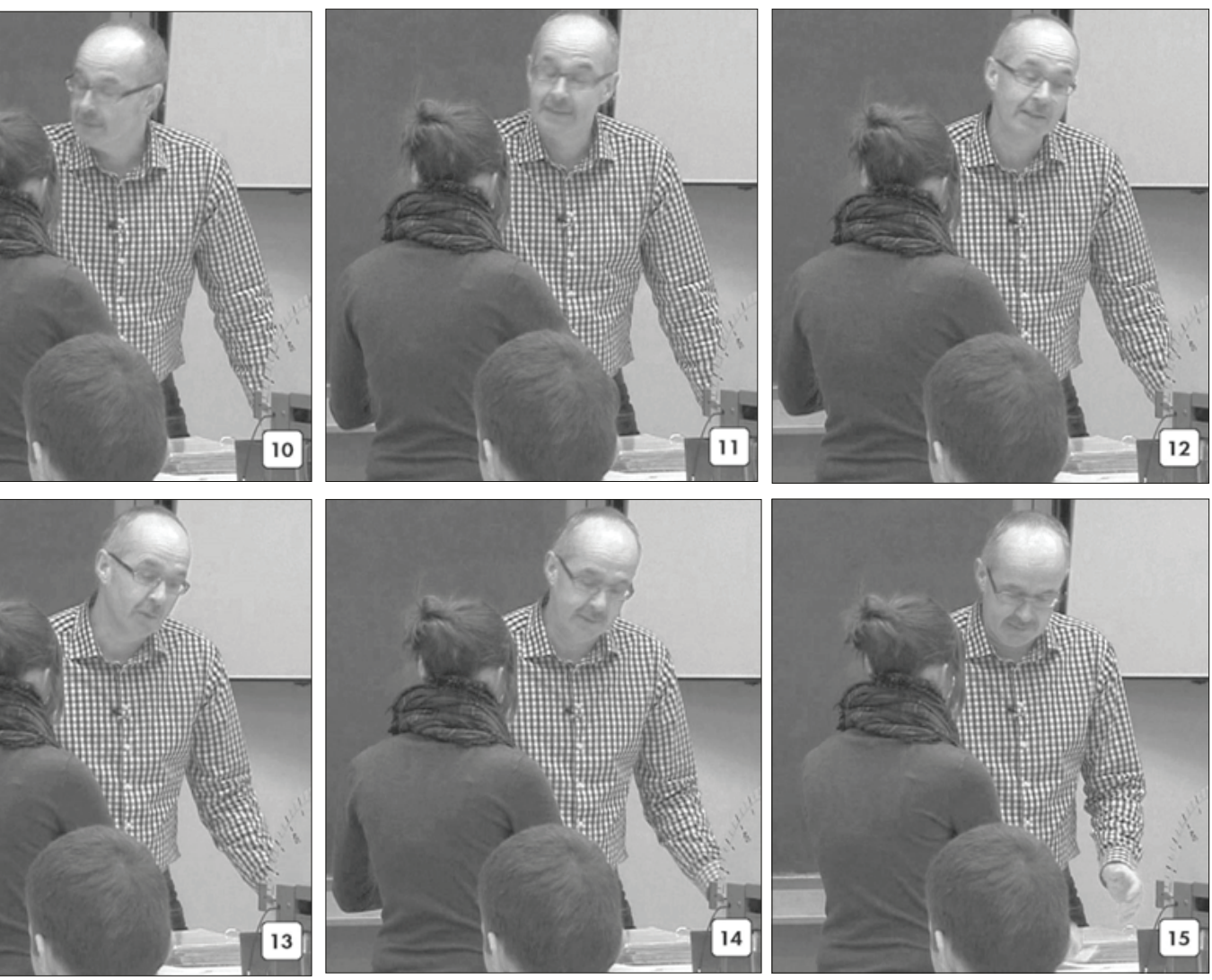

n die Kopfbewegung sequenzstrukturell nicht nur als second pair part sehen, auch als einen Hinweis darauf, dass Schüler und Lehrer in einer gemeinsamen reit agieren und ihre individuellen Handlungsrealisierungen mikrostrukturell eren. Eine offensichtliche Implikation der Kopfbewegung und der mimischen nsweise des Lehrers besteht in der lokalen Auflösung der blicklichen Orientieden Schüler: Er will/kann ihn aktuell nicht mehr sehen bzw. steht momentan für zur Verfügung.

ifische Expressivität und Markiertheit sowie die Unmittelbarkeit der lokalen Aufon availability bringen noch etwas anderes zum Ausdruck. Der Lehrer stellt in Ichen Deutlichkeit und Schnelligkeit Genervt-Sein zur Schau, kaum dass der zu lachen begonnen hat, dass dies keine ernsthafte Reaktion sein kann, es sei ist offen diskriminierend. Der Ausdruck seines körperlichen Verhaltens signalimehr ein deutliches „Was ist denn jetzt schon wieder“ oder „Nicht schon wieder“, hes alleine das situative Verhalten des Schülers (sein freudiger Eintritt in den aum) als Bezugspunkt inadäquat erscheint.

so die situativen Verhaltensweisen keine hinreichende Motivierung des Lehrerns liefern, muss man danach fragen, welche situationstranszendierenden Aspekte Verhalten verantwortlich sein können. Unter der Voraussetzung, dass der Lehrer em Verhalten verstehbar, situationssensitiv sowie interaktionsstrukturell und ch passend auf das Interaktionsangebot des Schülers reagiert, muss man weitern ausgehen, dass der Schüler hierfür über die notwendigen Wissens- und Versteaussetzungen verfügt. Er kann dazu jedoch nur auf vergleichbare, in der gemeinnteraktionsgeschichte liegende Verhaltensweisen des Lehrers ihm oder anderen 
er/innen gegenüber zurückgreifen. Und er kann die zwischen ihm und dem Lehrer hende Beziehung als relevant in Rechnung stellen. Dies und die interaktionsstruktuSpezifik des Lehrerverhaltens als auf sein Ködern reagierender Zug stellen also schütige Ressourcen dar, die es ihm gestatten, das Verhalten des Lehrers daraufhin zu gen, ob es eine Ratifikation seines Interaktionsangebotes und der damit verbundenen ktionsmodalität ist.

chüler nähert sich dem Lehrerpult weiter, wobei er seinen Blick weiterhin auf den r geheftet hat, der mit den Unterlagen beschäftigt ist. Dabei hat er seine rechte Hand rhin in die Seite gestützt, so als habe er Seitenstechen (Bild 16a, b).
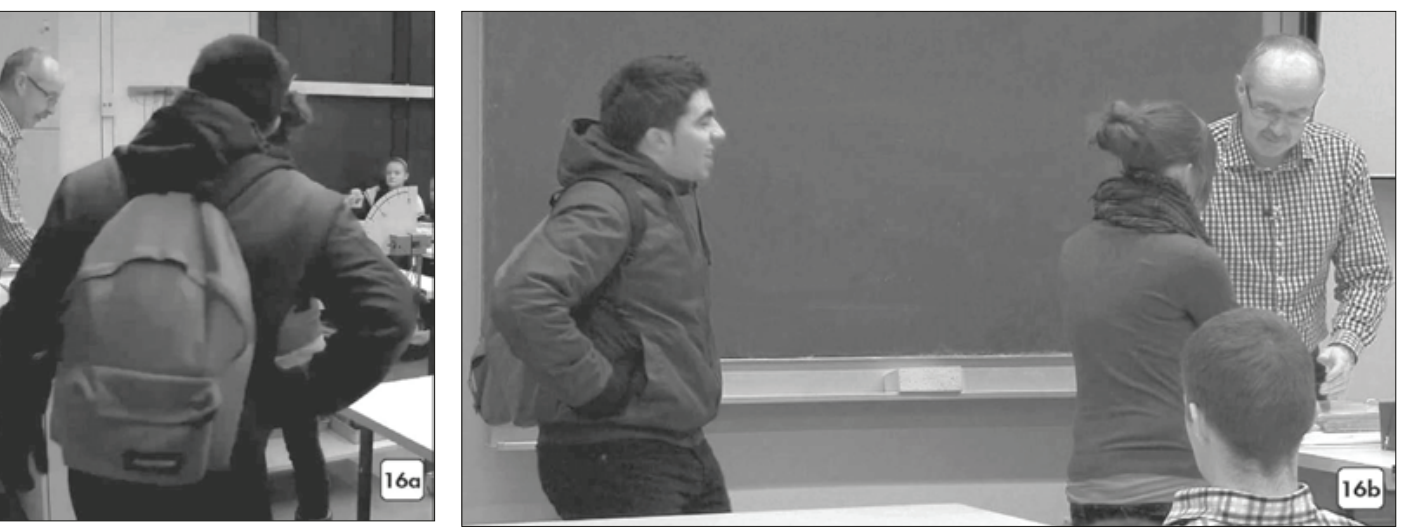

nstechen als Ausdruck und Folge körperlicher Anstrengung (im Ausdauerbereich) in der Regel mit anderen Ausdrucksformen einher, zu denen unter anderem auch k gehört (offener Mund). Der Gesichtsausdruck einer Person, die Seitenstechen hat, cherlich nicht das offene Lachen, das wir hier im Gesicht des Schülers sehen. Wir a es also mit einer widersprüchlichen und unstimmigen Ausdrucksfigur zu tun.

Simik des Schülers kontextualisiert das Seitenstechen als unernst und seine Präsenz samt als eine des ,so-tun-als-ob“. Die primäre interaktive Funktion dieser Haltung Produktion von Auffälligkeit. Letztlich kann man die gesamte körperliche Präsenz chülers in der Aufforderung zusammenfassen: „Frag mich, was los ist!“

Schüler hält dann in seiner Bewegung in Richtung Lehrerpult für einen kurzen ent inne, blickt dabei den Lehrer unverändert an. Das kurze Verharren des Schülers e Reaktion darauf, dass er die Aufmerksamkeit des Lehrers durch dessen Wegdrehen opfes verloren hat. Er behält das Lächeln so lange bei, bis der Lehrer sich ihm wieicklich zuwendet und dabei ebenfalls lächelt (Bild 17).

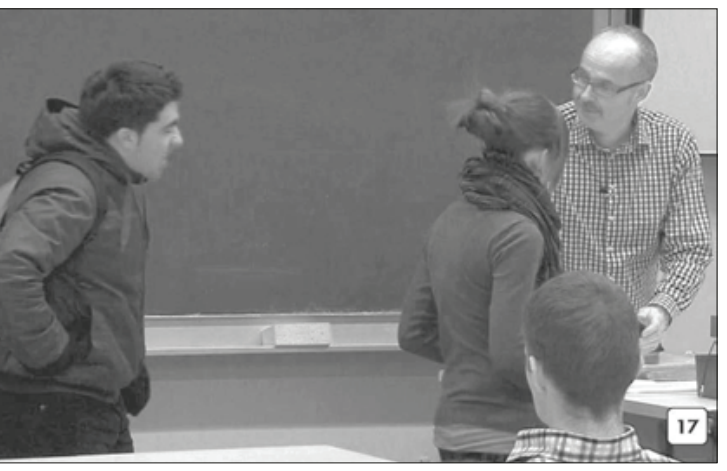


üler produziert Sichtbarkeit und Auffälligkeit zur Unterbreitung eines Interaktibots. Er setzt dazu auch die Widersprüchlichkeit unterschiedlicher Ausdruckswei- Seitenstechen und Lachen) ein, um eine spezifische, spielerische Interaktionsit zu offerieren. Passend zum Seitenstechen ist seine Verankerung am Lehrerpult, am besten mit Zusammensacken beschreiben lässt, wobei er sich mit beiden Händem Pult und dem Versuchstisch aufstützt (Bild 18).

alten des Schülers kontrastiert maximal mit dem der Schülerin. Dies gilt für seine anisation, seine Nähe-Distanz-Regulierung, Gehen als situierte Praktik (Schmitt ind seine auf fokussierte Interaktion ausgerichtete Präsenzfigur. Besonders deutd der Kontrast, wenn man die Positionen der Schülerin und des Schülers verdie sie am Lehrerpult einnehmen (Bild 3, 18).
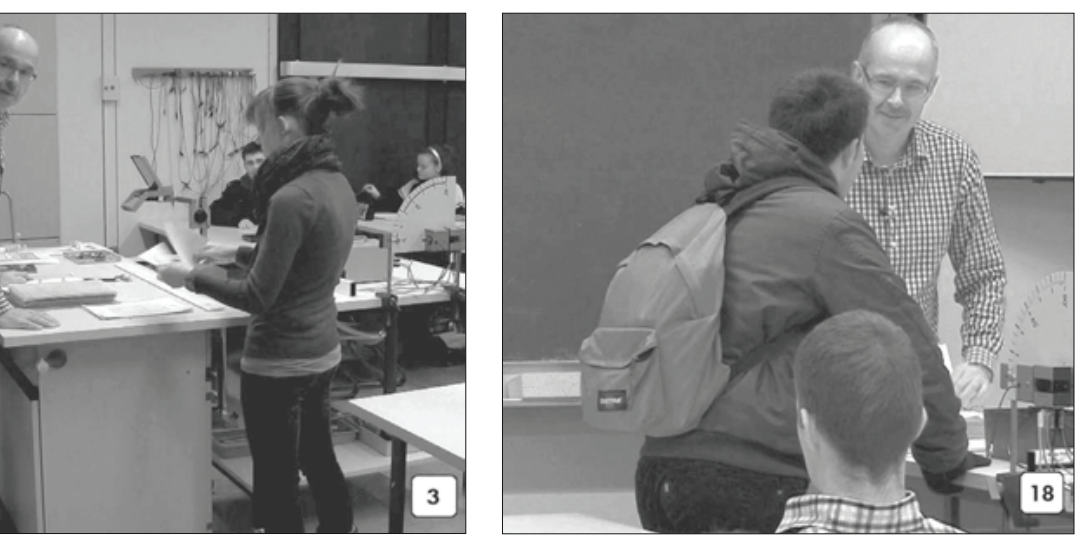

veise gleichzeitige Lachen/Lächeln ist ein Verhaltensmerkmal, das den Eindruck lt, es geht recht unterhaltsam zu in der gut koordinierten Dyade. Die Momente, in ch ihre Blicke treffen, die punktgenaue Organisation und Dynamik von Blickzukabwendung sind zudem Ausdruck einer weitgehenden Synchronisierung. ${ }^{10}$

te Timing der individuellen Verhaltensweisen hat seine Grundlage in einem gemeinhythmus und einer gemeinsamen Zeitlichkeit. Die Feinabstimmung und das Pashältnis beider Beteiligungsweisen weisen darauf hin, dass dies nicht erstmalig in tion, sondern im wechselseitigen Wissen um die Relevanz interaktiver Vorgängighieht. Dabei spielt die existierende Beziehung als soziale Trägerstruktur der aktuelaktion eine wesentliche Rolle. Sie ist die zentrale Voraussetzung dafür, dass der Im dem Schüler seine Orientierung auf das Ende der dyadischen Interaktion zu veren, diesen mit einem Klaps auf den linken Oberarm ,anschieben“ kann (Bild 19).

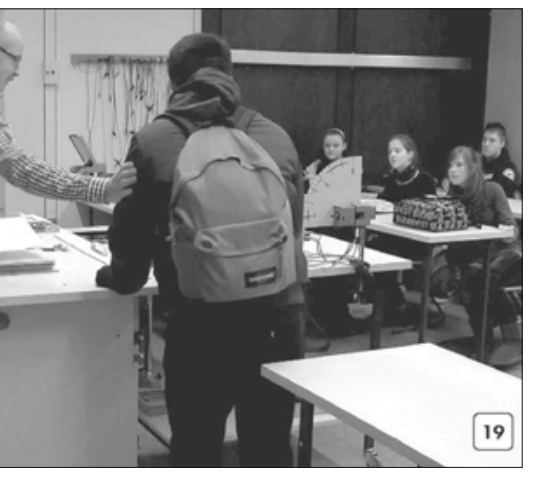

teraktiven Synchronisierung siehe Heidtmann/Schmitt (2010). 
nteraktion wird von Anfang an auch für die Klassenöffentlichkeit als ein gemeinsaUnternehmen der beiden Beteiligten vollzogen. Auch die Klassenöffentlichkeit nt die Spezifik der Beziehungsstruktur als Träger dieser Inszenierung zu erkennen. ndest liefert der Schüler einen klaren ,account“ für die wichtige Rolle der Klassentlichkeit. Unmittelbar nach Abschluss der Interaktion mit dem Rücken zum Lehrer nd streckt er mit einem Lachen im Gesicht die Zunge heraus (Bild 20a, b).
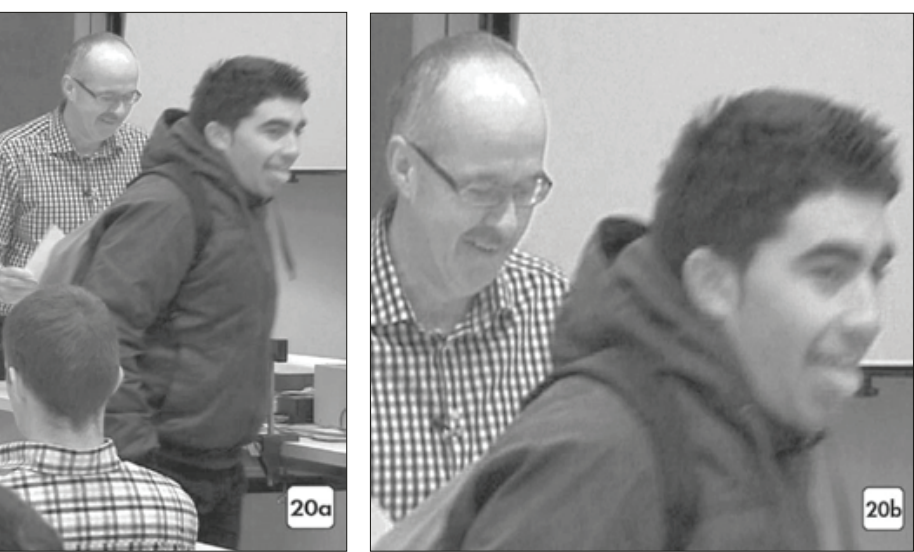

r „account“ setzt voraus, dass der Schüler unterstellt, die Klassenöffentlichkeit indest ein Teil davon) sei seiner Interaktion mit dem Lehrer soweit gefolgt, dass sie erausstrecken der Zunge in angemessener Weise als Kommentar der zurückliegenteraktion verstehen kann.

ehrer realisiert im Vergleich zu seinem Verhalten der Schülerin gegenüber mit seirörperlichen Verhalten ein eher nicht-kategoriales, individualisiertes recipient design. Verhalten reagiert nicht nur auf ,einen verspätet eintretenden Schüler“, sondern auch as spezifische Angebot, das dieser konkrete Schüler durch sein Verhalten unterbreiesentliche Merkmale der visuell wahrnehmbaren Seite des recipient design sind:

chnelligkeit der Reaktion,

Expressivität des Ausdrucks,

ounktuelle Reduktion von Availability bei gleichzeitigem Lachen als wesentlicher ndikator der Interaktionsmodalität und ein

unktueller körperlicher Kontakt.

Jemeinsamkeit der beiden unterschiedlichen recipient designs wird deutlich: Der rr reagiert sowohl auf die Selbstpräsentation der Schülerin (Verhaltenstransparenz) ch auf die des Schülers (funktionale Verhaltensintransparenz) bestätigend. Man kann laher fragen, ob das Verhalten des Lehrers als ein prototypischer Fall interaktiver ligten-Konstitution interpretiert werden kann. Für diesen Prototyp wäre die weitge, gleichsinnige Spiegelung eines Verhaltensangebots charakteristisch. ${ }^{11}$

gänzlich anderer Prototyp liegt in dem in Schmitt/Deppermann (2009) analysierten Fall vor. Dort spiet ein Dozent gerade nicht das von einem Studenten offerierte Verhaltensangebot „professionell Wisder", sondern dekomponiert dieses in analytischer Weise und repräsentiert den Studenten dadurch als vissend und anmaßend. 


\subsection{Verbalanalyse: Äußerungs- und Interaktionsstruktur}

Bei der visuellen Erstanalyse wird an verschiedenen Stellen deutlich, dass der interaktive Austausch nicht ohne Verbalität auskommt. ${ }^{12}$ Wenden wir uns nun der Analyse des verbalen Anteils der Interaktion zu und schauen, welche für unser Erkenntnisinteresse relevanten Einsichten sich dadurch eröffnen. Nachfolgend rekonstruieren wir die äußerungs- und interaktionsstrukturelle Spezifik des verbalen Austauschs ohne suprasegmentale Information. Bei der Präsentation der Ergebnisse der Verbalanalyse verzichten wir zudem auf die bei der visuellen Erstanalyse bereits produzierten Einsichten.

Minimaltranskription des Interaktionsausschnitts (nach Selting et al. 2009)

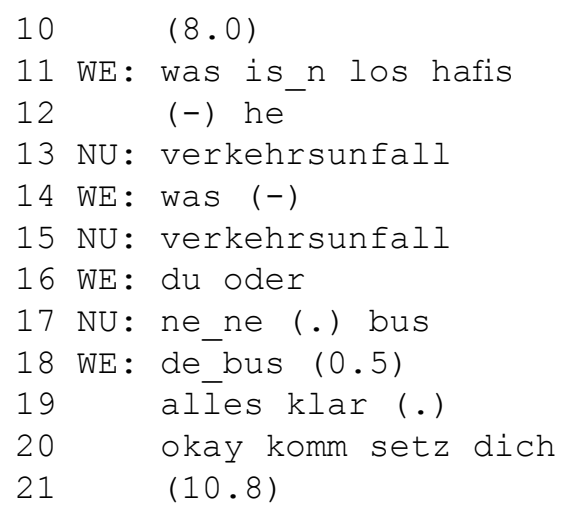

Das verbale Geschehen wird durch die Frage des Lehrers was is_n los hafis initiiert. Obwohl die Äußerung klar als Frage erkennbar ist, realisiert der Lehrer letztlich einen reaktiven Zug. Der Bezugspunkt seiner Reaktion liegt jedoch nicht in verbaler Form vor. Sequenzstrukturell reagiert der Lehrer mit seiner Thematisierung wahrscheinlich auf visuell wahrnehmbares Verhalten eines Beteiligten in der acht-sekündigen Pause, der mit hafis namentlich angesprochen wird. Hinsichtlich des Äußerungsformates kann man die Frage des Lehrers als Verstehensdokumentation bezeichnen.

An der Äußerung sind unterschiedliche Aspekte von Bedeutung. Das Fragepronomen was in Frontstellung setzt beispielsweise voraus, dass der Lehrer etwas wahrgenommen hat, was er mit dem Konzept ,etwas ist los“ fassen kann. Impliziert sind damit wiederum zwei unterschiedliche Aspekte: Zum einen wird präsupponiert, dass etwas los ist, zum anderen lässt das Konzept „etwas ist los“ Annahmen über die Qualität des Sachverhaltes zu, der mit was erfragt wird. Der präsuppositive Gehalt der Frage wird zudem durch die (hier verbenklitisch realisierte) Modalpartikel denn verstärkt, die die Frage als Schlussfolgerung des Lehrers aus dem vorgängig Wahrgenommenen ausweist (Deppermann 2009). Als epistemisch operativer „konklusiver Konnektor“ (ebd., S. 29) zeigt die Partikel den Beteiligten gleichermaßen an, dass die Frage durch den vorangehenden Kontext (des Zuspätkommens) veranlasst ist, und dass der für den Bezugskontext verantwortliche Schüler dessen Zustandekommen auch erklären kann.

12 Aus Sicht der visuellen Erstanalyse kann man sich also für die interaktionsstrukturellen und verhaltensspezifischen Aspekte sensibilisieren, die auf die „Wahrscheinlichkeit der Notwendigkeit zum Sprechen“ hindeuten. Es handelt sich dabei um die Umkehrung der Struktur, die der Vorstellung von einer „Rede begleitenden Gestik“ zugrunde liegt. Hier geht es um eine Form des „Verhalten begleitenden Redens“. 
Auch die nachgestellte, segmental nicht abgetrennte, namentliche Adressierung hafis ist implikativ. Hinsichtlich der Relevantsetzung steht für den Lehrer das wahrnehmbare Geschehen und nicht der Produzent bzw. seine Adressierung im Vordergrund bzw. äußerungsstrukturell ,an erster Stelle“ (Lerner 2003). ${ }^{13}$

Da wir es mit einer verbalen Interaktionsdyade zu tun haben, die in der Öffentlichkeit der Klasse agiert, kann man bezogen auf die Position der Adressierung eine Interpretation entwickeln, bei der entweder Mehrfachadressierung und/oder so etwas wie Aufmerksamkeitssteuerung für die anderen Schüler eine Rolle spielt. Die primäre Implikation des Lehrerverhaltens bestünde dann darin, das von ihm Wahrgenommene auch für die Wahrnehmung der Klassenöffentlichkeit als relevant anzubieten und Hafis als Urheber kenntlich zu machen. Seine Thematisierung bezieht sich jedoch auf bereits vergangenes Verhalten. Bezogen auf die Aufmerksamkeitssteuerung der anderen würde der Hinweis auf Hafis wahrscheinlich etwas zu spät kommen. Es sei denn, er besitzt projektive Qualität im Sinne von: Es wird auch noch nachfolgend etwas Wahrnehmenswertes geben, sei es die Verhaltenskonstanz von Hafis, sei es ein von ihm selbst oder gemeinsam produziertes „Angebot““.

Insgesamt sind mit der Wahrnehmungsthematisierung des Lehrers folgende Implikationen verbunden: Er thematisiert

a) ein (wahrscheinlich) nur visuell wahrnehmbares Verhalten,

b) das über eine gewisse Auffälligkeit/Qualität verfügt, wobei die Auffälligkeit entweder im Verhalten selbst begründet sein kann (Expressivität) oder in Aspekten des Unerwarteten (Auftauchen und Anwesenheit zum unerwarteten Zeitpunkt oder in ungewöhnlichen Kontexten [hier konkret zu spät kommen]),

c) das sich ihm (deswegen) zur Wahrnehmung und Thematisierung aufdrängt,

d) das in seinen interaktiven und sozialen Implikationen nicht evident ist,

e) das in seiner spezifischen Qualität auch für die Wahrnehmung durch die Öffentlichkeit bestimmt ist,

f) für das der mit hafis Angesprochene entweder ursächlich verantwortlich gemacht oder als jemand angesprochen werden kann, der zur Erklärung dieser Implikationen beitragen kann und

g) für den mit der Wahrnehmungsthematisierung eine deutliche Reaktionsverpflichtung relevant gemacht wird.

Nach einer kurzen Pause hängt der Lehrer an seine Wahrnehmungsthematisierung die Nachfrage he an. Das he hat nicht die Funktion, die Pause, die sie segmental von der Frage abtrennt, nachträglich als ,transition relevance place“ auszuweisen und dem Schüler damit zu verdeutlichen, dass er die erste Möglichkeit zur Reaktion hat vorübergehen lassen. Dem he kommt vielmehr im Lichte der gerade entwickelten Interpretation, bei der die Wahrnehmungsstrukturierung der Klassenöffentlichkeit eine dominante Rolle spielt, eine wichtige Bedeutung zu. Es indiziert die nunmehr ausschließliche Orientierung des Leh-

13 Lerner (2003, S. 185) betont: „Post-positioned terms of address are regularly employed as a device to demonstrate a particular stance toward or relationship with a recipient under circumstances where that demonstration is particularly relevant. [...] it upholds the (already adequately established) intended recipient. As such, it underlines the very act of speaking expressly to the already addressed recipient. Moreover, to use an address term is always to say something about the addressed party." 
rers auf den Schüler und betont damit die primär dyadische und personen- und beziehungsspezifische Qualität dieser Orientierung.

Bei der Interaktionsstruktur wird insgesamt ein Spannungsverhältnis deutlich zwischen der expliziten und hochgestuften Wahrnehmungsthematisierung des Lehrers (was is_n los hafis (-) he, Zeile 11-12) und deren anschließenden Bearbeitung. Der Schüler reagiert auf Fragen und Nachfragen jeweils mit einem maximal reduzierten Äußerungsformat, das zudem zwischen Assoziationsreichtum und Verschleierung oszilliert. Da auch der Lehrer immer rasch mit weiteren Nachfragen reagiert, entsteht eine kleinschrittige, kurz getaktete Interaktionsdynamik (siehe besonders Zeile 13 bis $18 \mathrm{im}$ Transkript).

Der Schüler verfolgt mit seinem verknappten Format ein Verfahren des „Köderns“ und überlässt es dem Lehrer, die Interaktion durch Fragen zu strukturieren. Er gibt auch auf Nachfrage kaum etwas preis, was es dem Lehrer etwa ermöglichen würde, die Relevanz des Verkehrsunfalls adäquat einzuschätzen. Er liefert jedoch gerade dadurch Hinweise darauf, worum es (ihm) eigentlich geht: So wie er nicht wirklich Seitenstechen hat, scheint es auch nicht wirklich um den Verkehrsunfall zu gehen. Der ist letztlich nur thematisches Beiwerk im Gesamtkonzept der spielerisch-performativen Bearbeitung seines Zu-spät-Kommens.

Bezüglich des recipient design lässt sich festhalten: Der Lehrer realisiert (auch) mit seinen verbalen Aktivitäten ein individualisiertes recipient design, das speziell auf Hafis zugeschnitten ist. Wesentliche Konstituenten des äußerungsstrukturellen recipient design sind Indirektheit/Implizitheit der Informations-Prozessierung und pragmatischen Verdeutlichung und damit die Etablierung eines gemeinsamen Inferenzrahmens.

\subsection{Analyse der Prosodie}

Wir haben auch bei der äußerungs-und interaktionsstrukturellen Analyse Hinweise darauf erhalten, dass der Austausch markiert ist. Wir sind jedoch nicht in der Lage, diese Markiertheit auch „sozial zu lesen“. Wir wissen nicht, in welcher Modalität die beiden Beteiligten den Eintritt in die Klasse bearbeiten: Ist sie ernsthaft, aggressiv, spielerisch? Eine Antwort erhoffen wir uns von der Analyse der stimmlichen und suprasegmentalen Aspekte des verbalen Austauschs.

Prosodie ist ein Mittel der Auffälligkeits- bzw. Kontrastproduktion und damit zur Inferenzgenese. Diese Funktion wird in der Wahrnehmungsthematisierung des Lehrers (in Zeile 11) besonders deutlich, bei der die prosodische Markierung nicht der informationsorientierten Binnenstrukturierung dient, sondern der Schaffung eines globalen Fokus auf die Äußerung.

Markiertheit bzw. die globale Fokussierung entsteht einerseits durch die Pitchkontur, die nach frühem treppenartigem Anstieg bei, was is “ plateauartig auf relativ hohem F0-Niveau mit geringem Range (zwischen 220 und 190 Hz) verläuft; andererseits wird Markiertheit durch das Alignment von Pitch und Intensitätsstruktur produziert, d.h. intonatorische Prominenz und Druckakzente sind tendenziell komplementär verteilt. Daneben sorgt segmentale Dehnung im langen /o/ von , los 6 , im eigentlich kurzen $/ I /$ und im finalen $/ s /$ von ,Hafis‘ für Markiertheit (Grafik 1). 


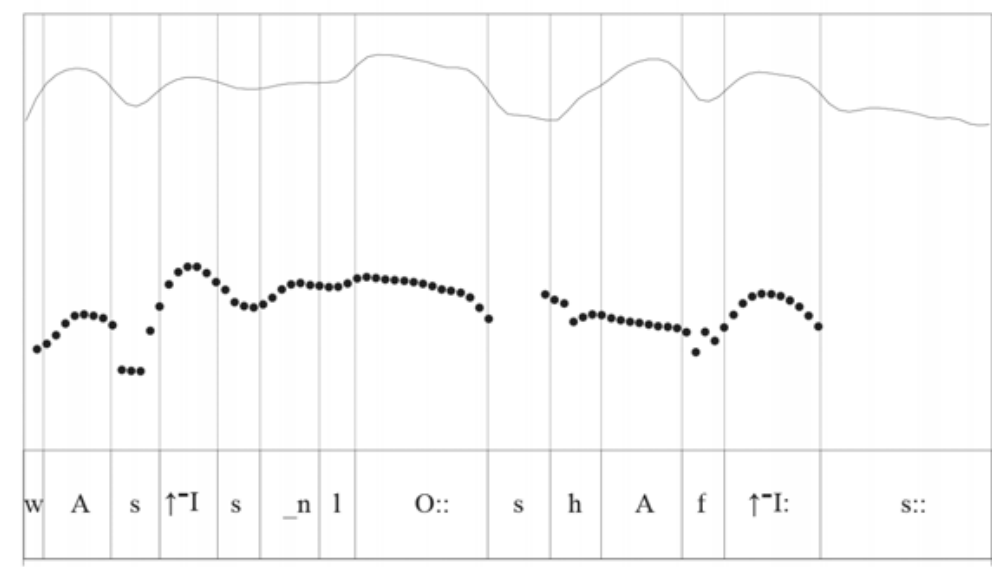

Grafik 1: Intensitätskontur (oben) und Pitchkontur (unten) der Wahrnehmungsäußerung

Eine klare artikulatorische Zäsur besteht zwischen der Adressierung und nachfolgender Fragepartikel he (in Z. 12) durch Pause (400ms Dauer) und durch prosodischen Kontrast. Der prosodische Kontrast konstituiert sich einerseits durch einen Intensitätskontrast und andererseits durch F0-Kontrast, den man als Wechsel in ein höheres und dynamischeres Intonationsregister bezeichnen kann (F0-Range zwischen $180 \mathrm{~Hz}$ und $330 \mathrm{~Hz}$ ).

Diese Zäsur ist in zweifacher Hinsicht funktional. Zum einen indiziert der Wechsel vom akzentkumulativen Register der Wahrnehmungsthematisierung in das leisere und höhere Register die Umorientierung des Lehrers von der Mitadressierung der Klasse hin zur primär dyadischen Interaktion mit dem Schüler. Zum anderen wird das kontrastiv markierte he zum nachgelieferten Ironiemarker, der die spielerische Modalität der vorgängigen Wahrnehmungsäußerung reflektiert. Die unterbrochene spielerische Modalität wird im weiteren Verlauf der kurzgetakteten Interaktion wieder prosodisch unterstützt aktualisiert, und zwar insbesondere durch saliente steig-fallende Akzente auf gedehnten Nuklei in den Nachfragen $<<\mathrm{f}>{ }^{\wedge} D U:>(Z .16)$ und $<<\mathrm{f}>$ de_${ }_{-}^{\wedge}$ BU:S? $>$ (Z. 18), siehe Graphik 2 und 3.
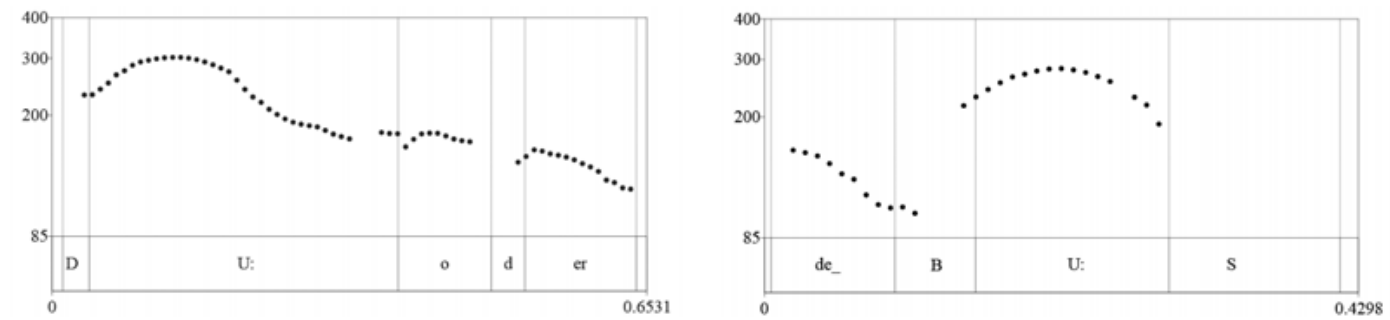

Grafik 2 und 3: Steig-fallende Pitchkonturen auf $D U$ und $B U S$

Zusammenfassend lässt sich bezüglich der im Ausschnitt gebrauchten prosodischen Ausdrucksressourcen festhalten, dass diese sowohl organisatorisches als auch modalisierendes Indexikalisierungspotenzial einbringen. Zum einen schafft die prosodische Gestaltung der Lehrerbeiträge Aufmerksamkeit und hat adressatensteuernde Wirkung, indem sie der interaktiv nicht direkt beteiligten Klassenöffentlichkeit ein Wahrnehmungsangebot macht. Die Mitadressierung der Klasse wird in nuce an der prosodischen Einbettung des an sich markierten Adressierungsterms $h A f \uparrow I: s$ in die saliente übergeordnete Struktur der Wahrnehmungsthematisierung deutlich, deren Wirkung man zugespitzt als ,,auftrittseinleitend“ bezeichnen kann. 
Zum anderen trägt Prosodie dazu bei, die thematisch-pragmatische Interaktion sozial verstehbar zu machen; ein Inferenzpotenzial der prosodischen Markierung besteht in der Anzeige der primär spielerischen Interaktionsmodalität und damit letztlich auch darin, die Vertrautheit der Interagierenden mit der sich entwickelnden Situation und der Qualität der Beziehung zwischen den Beteiligten als Grundlage ihrer (spielerischen) interaktiven Gestaltung anzuzeigen. Die modalitäts- und beziehungsindikative Funktionalität von Prosodie kann in der Begrifflichkeit von Gumperz (1982, S. 130ff.) als Kontextualisierung bezeichnet werden.

Als Kontextualisierungshinweis wirkt prosodische Markierung neben anderen Kontextualisierungshinweisen - und zwar besonders neben indexikalischen Mitteln verschiedener nicht-auditiver Ausdrucksebenen. Prosodische und visuelle indexikalische Zeichen bilden als Kontextualisierungsmittel, die im Interaktionsausschnitt teilweise koordiniert und komplementär, teilweise redundant distribuiert sind, die Grundlage der Genese sozial relevanter Bedeutung und ihrer Interpretation.

In prosodischer Hinsicht realisiert der Lehrer ein doppeltes recipient design. Hafis wird dabei als individueller Schüler positioniert, der über eine gemeinsame Interaktionsgeschichte mit und eine spezifische Beziehung zum Lehrer verfügt. Beides ist Voraussetzung des spielerischen interaktiven Umgangs und der indirekten Bearbeitung des Zu-spätKommens. Die Klassenöffentlichkeit wird als Publikum eingeladen, der situativen Performance der Dyade zu folgen.

\section{Fallanalytisches Resümee: Die Komplexität der Ausdrucksressourcen}

Unsere Fallanalyse hat verdeutlicht, dass sehr unterschiedliche Ausdrucksressourcen an der Produktion des recipient design des Lehrers beteiligt sind. Wir wollen nachfolgend die einzelnen Ausdrucksressourcen in ihrem Zusammenspiel darstellen und dabei insbesondere die je eigene Zeitlichkeit, in der die einzelnen Ressourcen zum Einsatz kommen, aufzeigen. Ziel dieses Überblicks ist es, aus extrakommunikativer Perspektive Einsichten in die faktische Komplexität des recipient design zu eröffnen, die wir im Handlungsvollzug oft auf die uns dominant erscheinende Ausdrucksressource „Verbalität“ reduzieren. Der tatsächliche ressourcenspezifische und sequenzielle Zusammenhang zeigt, dass die Produktion des recipient design ein zeitlich gestreckter Prozess ist, in dem vorgängige Ausdrucksressourcen nachfolgende projizieren und dadurch unter anderem die „Autonomie des Verbalen“"stark einschränken.

Das nachfolgende Modell (Grafik 4) ist zudem eine geeignete Grundlage, um aus den darin festgehaltenen fallspezifischen Aspekten generelle Fragen für die Entwicklung eines multimodalen Konzeptes von recipient design zu entwickeln und offene Punkte und Probleme zu identifizieren (Kap. 7).

Im Modell sehen wir das genaue zeitliche Alignment der auf der Y-Achse gelisteten Ausdrucksressourcen ab dem Zeitpunkt des Türöffnens durch den Schüler bis zum Ende der Wahrnehmungsthematisierung. Start, Dauer und Endpunkt der einzelnen Aktivitäten sind in Bezug auf die Timeline notiert (in Sekunden und Millisekunden).

Deutlich wird die konkomitant prosodisch und mimisch-gestisch generierte Prominenz der Wahrnehmungsthematisierung, insbesondere die saliente Kopfdrehung mit begleitender Mimik. Aus der detaillierten Rekonstruktion des zeitlichen Zusammenhangs der an 
Konstitution des multimodalen recipient design beteiligten Ausdrucksressourcen sich folgende Einsicht: Neben der kontextualisierenden Funktion von Prosodie für le Äußerungen (an die sie produktionstechnisch gebunden ist) scheint es auch eine xtualisierende Beziehung zwischen einzelnen Ausdrucksressourcen zu geben. So en visuell wahrnehmbare Aspekte des Verhaltens spezifische verbale und prosodiRealisierungen projizieren und festlegen.

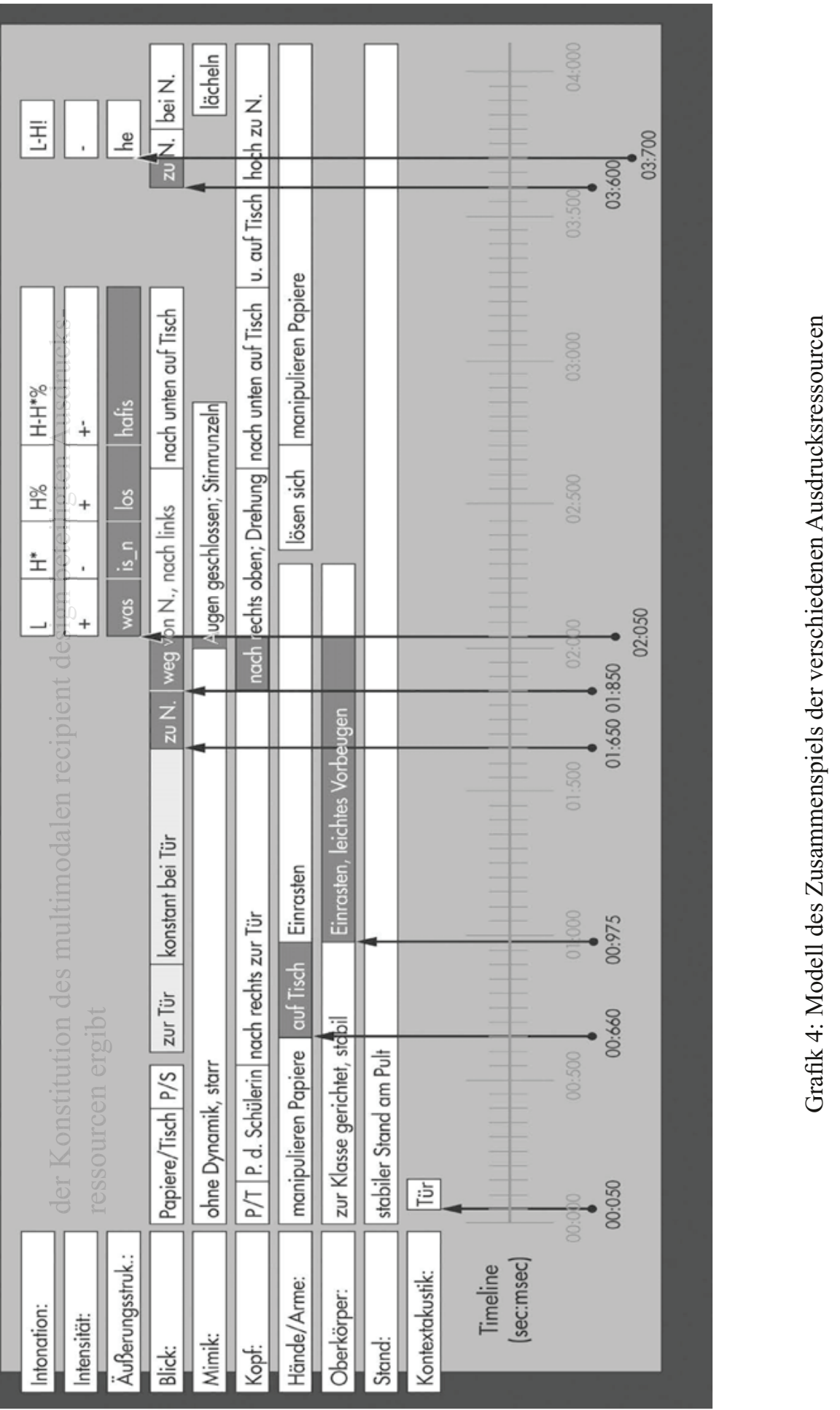


Am Modell der Ausdrucksressourcen sehen wir, dass der Verbaläußerung projizierende Veränderungen im visuell wahrnehmbaren Ausdruck vorausgehen: Da ist insbesondere

- das Absetzten der Hände auf dem Pult (t: 00:660),

- das Einrasten der Positur (t: 00:975),

- der konstante Blick zur Tür, der zum Blick zum Schüler wird (t: 01:650) und sich kurz vor der Verbaläußerung von ihm wieder wegbewegt (t: 01:850),

- der Blick zum dann primär adressierten Schüler (t: 03:600), der dem prosodischen Registerwechsel in der verbalen Äußerungseinheit 100ms vorausgeht.

Man kann erkennen, wie der Lehrer - im Bemühen, ein Maximum an interaktiver Beteiligung zu organisieren - seine Orientierung auf den Schüler und die Klassenöffentlichkeit sequenziell und alternierend organisiert. Verbalität und Prosodie werden dabei sequenziell nachgeordnet eingesetzt, um die Unterrichtsöffentlichkeit auf das Geschehen in der Dyade aufmerksam zu machen und die Wahrnehmung der übrigen Schüler zu strukturieren. Während das visuell wahrnehmbare Verhalten des Lehrers nur Beteiligte erreicht, die im Moment des Verhaltensvollzuges diesen zufällig sehen, richtet sich Prosodie, nachdem die Interaktionsdyade visuell wahrnehmbar bereits konstituiert ist, auch an diejenigen, die hinsichtlich ihrer visuellen Wahrnehmung anders orientiert sind.

Das komplexe recipient design trägt also in unserem Fall aktiv zur Organisation interaktiver Beteiligung in der Dyade und zur Verdeutlichung der Klasse als relevantes Interaktionsensemble bei (Schmitt/Deppermann 2007; Schmitt 2012c; Schmitt 2013, Kap. 6).

\section{Multimodales recipient design: Offene Punkte}

Bei dem Versuch, die Orientierung eines Interaktionsbeteiligten auf andere Beteiligte und seine Sensitivität für bestimmte Beteiligte als relevantes und empirisch salientes Phänomen interaktionstheoretisch zu rahmen, stellt die konversationsanalytische Vorstellung recipient design generell einen relevanten Bezugspunkt dar. Angesichts unserer fallspezifischen Ergebnisse zeichnen sich jedoch eine Reihe offener Punkte ab, die bei der multimodalen Adaption des Konzeptes zu beachten sind. Hierzu gehören (u.a.):

- das Mitgebrachte und das Erzeugte (,,brought along“ und „brought about“) (7.1),

- Adressatenvielfalt/-problematik (7.2),

- die strukturelle Reflexivität (7.3),

- die Aktivitätssensitivität (7.4),

- die strukturelle und formale Diffusität (7.5),

- die Einheitenkonstitution (7.6),

- die Benennung (7.7),

- die theoretische Neu-Kontextualisierung (7.8),

- die Handlungsfunktionalität/Rhetorische Qualität (7.9). 
7.1 Das Mitgebrachte und das situativ Erzeugte

(,,brought along“" und „brought about")

Das Verhältnis von „brought along“ und „brought about" ${ }^{“ 14}$ verweist auf das Spannungsverhältnis, das durch folgende Frage charakterisiert wird: Steht das recipient design bereits zu Beginn fest, oder entsteht es erst in der Interaktion in rekonstruierbarem Bezug auf vorgängiges Interaktionsgeschehen?

Man muss sich die Dichotomie von „brought along“ und „brought about“ als theoretisch gesetzte Endpunkte eines Kontinuums vorstellen. Bei der fallspezifischen Analyse wird man es daher immer mit einer spezifischen Gewichtung beider Aspekte zu tun haben. Dies ist evident in Fällen, in denen die aktuelle Interaktion auf der Grundlage relevanter interaktiver Vorgängigkeit konstituiert wird und bei denen die Beteiligten auf etablierte Beziehungen zurückgreifen können (Lehrer und Schüler/in).

Aber auch in Situationen des Erstkontaktes werden Orientierungen auf das Gegenüber relevant, welche auf interaktiv vorgängige Erfahrungen der Beteiligten beruhen. Es ist fraglich, ob es überhaupt einen Zeitpunkt zu Beginn einer fokussierten Interaktion gibt, wo das recipient design tatsächlich ,auf null gestellt“ ist und dann sukzessive und ausschließlich durch aktuelles, situatives Verhalten des Gegenübers bestimmt wird.

Das erzwingt die Differenzierung des „brought along“ hinsichtlich

a) solcher Wissensbestandteile und „Vor"-Einstellungen, die auf interaktiver Vorgängigkeit beruhen, welche die beteiligten Personen tatsächlich im persönlichen Kontakt miteinander gemeinsam haben und

b) hinsichtlich solcher, die nicht personell real vorliegen, jedoch aufgrund bestimmter Verhaltensaspekte der Beteiligten in kategorialer Form aktiviert werden.

Schmitt/Deppermann (2010) analysieren einen Fall, der ein prototypisches Beispiel für ein recipient design ist, das primär „brought about“ ist. Es steht im Zusammenhang mit Vermittlungsbemühungen eines Dozenten in einer Pitching-Sitzung (Heidtmann 2009). Nachdem ein Student mehrfach auf eher argumentativ-abstrakte Vermittlungsbemühungen des Dozenten bezüglich eines dramaturgischen Strukturbegriffs uneinsichtig reagiert hat, wählt der Dozent für seine Vermittlungsbemühungen eine neue Darstellungsweise, die durch Narration, Deskription, Performance und didaktisches Nachfragen gekennzeichnet ist.

Es handelt sich dabei um ein recipient design im Rahmen einer bereits seit längerer Zeit laufenden Interaktion. Man kann sehen, wie es in einem länger gestreckten Prozess im Verlaufe der Interaktion schrittweise entsteht. Es wird aufgrund seiner kontrastiven Struktur zum vorherigen Verhalten des Dozenten als etwas Neues deutlich und verweist klar auf den Ursprung seines Entstehens.

In dem von uns analysierten Beispiel liegt das gänzlich anders, da es keinerlei rekonstruierbare interaktive Vorgängigkeit gibt und das recipient design sofort - quasi aus dem Stand - aktiviert wird.

14 Die Vorstellung von „,brought along“ wurde von Hinnenkamp (1989) vorgestellt, der seinerseits auf Giddens (1976) referiert; siehe auch Auer (1992, S. 26), der „brought along“-Aspekte des Kontextes im Kontrast zu „brought about“-Aspekten differenziert. 


\subsection{Adressatenvielfalt/-problematik}

Unser Fall macht sicht- und hörbar, dass es zwei unterschiedliche Rezipienten gibt: der Schüler und die Klassenöffentlichkeit. Es ist also analytisch zu klären, inwieweit die Tatsache, dass für den Lehrer die Klassenöffentlichkeit ein relevanter Rezipient ist, dazu führt, dass er den Schüler als einen spezifischen Rezipienten mit Unterhaltungswert und Wahrnehmungsrelevanz konstituiert. Dabei muss man von einer dialektischen Beeinflussung beider recipient designs ausgehen und dies konzepttheoretisch reflektieren. ${ }^{15}$

In Mehrpersonenkonstellation und auf der Grundlage audiovisueller Interaktionsdokumente ist die Frage, wer Rezipient eines multimodalen Designs ist bzw. wer alles als Adressat im recipient design repräsentiert ist, weder banal noch evident. In unserem Fall bezieht sich die Problematik auf die Frage, inwieweit auch oder gerade die Klassenöffentlichkeit nicht der eigentliche Rezipient des Lehrerverhaltens ist. Denn grundsätzlich verdeutlicht der Lehrer durch seine Reaktion auf das initiative Verhaltensangebot des Schülers sowohl ihm, auf den er reagiert, als auch denjenigen, die darüber hinaus seine Reaktion wahrnehmen können, a) wer aus seiner Sicht Rezipient ist, b) wie er zu ihm steht und c) als was/wen er ihn der öffentlichen Wahrnehmung anbietet.

In der Interaktionsdyade ist es gänzlich unproblematisch, für wen ein spezifisches recipient design produziert wird und für wen die damit implizierten Informationen gedacht sind, da es nur den anderen als Rezipienten gibt. Das ändert sich jedoch grundlegend, wenn man analytisch in Mehrpersonenkonstellationen eintritt. Dann entsteht neben der Rekonstruktion der Bestandteile eines recipient design und der damit verbundenen Fremd- und Selbstpositionierung auch die Frage, für wen die damit zusammenhängenden Informationen gedacht sind. Für die multimodale Analyse bedeutet das, die einzelnen ausdrucksspezifischen Konstituenten eines recipient design genau auf diese Frage hin zu beleuchten.

Dies gilt insbesondere in Situationen, die wie der Schulunterricht durch eine stabile und von allen Beteiligten geteilte Wahrnehmungs-Wahrnehmungs-Struktur bestimmt werden. Dieser Punkt verschärft sich, wenn der Raum auf Wahrnehmung bestimmter Bereiche (das ,Vorne“ im Unterricht) und bestimmter Personen, die sich in diesem Bereich aufhalten (Lehrer als Fokuspersonen) interaktionsarchitektonisch ${ }^{16}$ ausgerichtet ist. Institutionelle Fokuspersonen wie Lehrer/innen wissen, dass sie kontinuierlich Objekte von Wahrnehmung sind, und nutzen dieses strukturelle Element für die Organisation des Unterrichts. Auf der Grundlage dieser stabilen Wahrnehmungs-Wahrnehmungs-Struktur realisieren sie systematisch doppelte recipient designs bei der Bearbeitung der strukturellen Anforderung, Kollektiv- und Individualorientierungen gleichzeitig zu bearbeiten (Schmitt, erscheint). Im Rahmen einer multimodalen Arbeitsteilung setzen sie dabei bestimmte Ausdrucksressourcen für den einen und andere Ausdrucksressourcen für den/die anderen Rezipienten ein.

\subsection{Strukturelle Reflexivität}

Betrachtet man das Konzept „recipient design“ hinsichtlich seiner Positionierungsimplikationen, dann sollte man diese - deutlicher als es bei der konversationsanalytischen Vorstellung zum Ausdruck kommt - bi-direktional interpretieren: Der eine Interaktionsbetei-

15 Dieser Zusammenhang ist aufgrund seines Erkenntnispotenzials zu wertvoll, um ihn einfach im „Container" der Mehrfachadressierung verschwinden zu lassen.

16 Zum Konzept „Interaktionsarchitektur“ siehe Hausendorf/Schmitt (2013). 
ligte repräsentiert durch sein recipient design nicht nur sein Gegenüber in der Interaktion in der Weise, dass er ihn/sie in interaktiv und sozial relevanter Weise positioniert. Der „Designer" positioniert sich selbst in unmittelbar vergleichbarer Weise und macht auch sich selbst - vielleicht sogar in erster Linie - als spezifischen Teilnehmer mit bestimmten sozialen, kulturellen und interaktiven Besonderheiten sichtbar. Man kann, wenn man dies möchte, recipient design in ein doppeltes bzw. duales participant design ,übersetzen“", um die Bi-Direktionalität der Positionierung auch terminologisch zu repräsentieren.

Dieser Aspekt der strukturellen Reflexivität (im Sinne eines dualen Designs) ist die zentrale Grundlage dafür, aus dem Verhalten eines Beteiligten ein Beziehungsangebot für den/ die Rezipienten rekonstruieren und dieses für die Analyse von Beziehungskonstitution nutzen zu können. So wie immer vier Finger auf den Zeigenden selbst gerichtet sind, wenn er auf einen anderen Beteiligten zeigt, so verweist auch die Art und Weise, wie er andere Beteiligte durch sein Verhalten positioniert, auf ihn selbst.

Es wäre daher konsequent, auf basaler Ebene nur von einem participant design zu sprechen und dieses jeweils in seiner analytisch dominant gemachten Positionierungsimplikation durch den Zusatz fremdbezogen oder selbstbezogen zu qualifizieren. Auch wenn eine solche Begriffsbildung terminologisch etwas holprig daherkäme, wäre sie hinsichtlich ihrer Positionierungsimplikationen analytisch transparent.

\subsection{Aktivitätssensitivität}

Ein weiterer offener Punkt ist die Frage nach den aktivitätsspezifischen Implikationen. Wenn es sich bei unserem Fall nicht um idiosynkratische Relevanzen handelt, muss man auf der Grundlage vergleichbarer Beispiele, für die ein Wechsel des Aktivitätszusammenhangs charakteristisch ist, klären, inwieweit die Sensitivität der Repräsentanz des Gegenübers nur auf diesen allein oder auch auf aktivitätsspezifische Bedingungen und Relevanzen reagiert.

Unsere Analyse hat gezeigt, dass es die jeweilige aktivitätsspezifische Umgebung ist, die es der Schülerin und dem Schüler erlauben, sich auf der Grundlage einer Verhaltenstransparenz (Schülerin mit unterrichtsbezogenem Handlungsziel in der Vorphase des Unterrichts) und einer funktionalen Verhaltensintransparenz (Schüler mit Spielangebot im Kontext eines Ordnungsverstoßes) zu präsentieren. Die Tatsache, dass der Lehrer in beiden Fällen diese Selbstpräsentation spiegelt, basiert auf der konvergierenden Orientierung der Beteiligten an den Relevanzen des jeweiligen Aktivitätszusammenhangs.

Man kann fragen, inwieweit es sinnvoll ist, von einer aktivitätsspezifischen „Normalform" des recipient design auszugehen. Damit würde die Sensitivität der Repräsentanz des Gegenübers im eigenen Verhalten unmittelbar an den dominanten Aktivitätszusammenhang rückgebunden und dieser als einer der wesentlichen Kontextbedingungen des recipient design konzepttheoretisch integriert.

Wenn man die Frage nach der konzeptuellen Relevanz aktivitätssensitiver Implikationen mit ja beantwortet, führt das auch zur analytischen Aufgabe, diese Implikationen von den adressatenspezifischen zu unterscheiden. Dies wäre dann im multimodalen Erkenntniszusammenhang eine vergleichbar analyseleitende Frage wie die Suche nach der empirisch basierten Differenzierung unterschiedlicher Rezipienten (7.2). Analysefaktisch führt das weiterhin zur Frage: Gibt es eine sequenzielle Abfolge, in der Aspekte, die auf die Aktivi- 
tätsstruktur reagieren, und solche, die der kategorialen oder individualistischen Ausstattung bzw. Unterstellung des Gegenüber gestiftet sind, realisiert werden.

Grundsätzlich würde das Konzept durch die Integration der Aktivitätssensitivität als konstitutive Realisierungsbedingung von recipient design seine Fixierung auf den Adressaten als dominante Orientierung bei der Rekonstruktion von Verhaltensspezifik ein Stück weit verlieren.

Zur falltranszendierenden Validierung unserer Ergebnisse bietet sich eine zweifache minimale Kontrastierung an: Zum einen kann man Interaktionen des Lehrers mit anderen zu spät kommenden Schülern untersuchen, zum anderen Interaktionen zwischen dem Lehrer und dem Schüler in anderen Unterrichtskontexten. Eine solche Kontrastierung wird insbesondere Hinweise auf den Zusammenhang zwischen aktivitätsbezogenen und beziehungsbezogenen Implikationen des recipient design produzieren.

\subsection{Strukturelle und formale Diffusität}

Die Rekonstruktion des Zusammenspiels der bei der Realisierung des lehrerseitigen recipient design beteiligten Ausdrucksressourcen, die im Modell die komplexe Qualität im Vergleich mit einem monomodal-verbalen Konzept verdeutlicht, pointiert die Frage nach der Nützlichkeit der monomodalen Vorstellung für die multimodale Interaktionsanalyse. Bei der multimodalen Adaption des Konzeptes muss nicht nur die in unserer Analyse deutlich gewordene segment-interne, kontextualisierende Beziehung zwischen einzelnen Ausdrucksressourcen integriert werden. Es muss auch ein Weg gefunden werden, mit den offenen Rändern der empirischen Grundlagen produktiv umzugehen (siehe 7.6).

Im multimodalen Erkenntniszusammenhang erweitert sich aufgrund der audiovisuellen Interaktionsdokumente die Vielfalt von Ausdrucksformen, die als mögliche Bestandteile eines recipient design in Frage kommen können. Die Entscheidung, welche Verhaltensaspekte im Einzelfall für das recipient design tatsächlich eine Rolle spielen, kann oftmals nicht „oberflächennah“ gefällt werden, sondern bedarf bereits analytischer Investigation. Das macht die multimodale Untersuchung von recipient design nicht gerade einfach.

Gibt man bei der empirischen Begründung die bislang praktizierte fraglose Fokussierung auf die verbale Ausdrucksressource auf, entsteht die Möglichkeit, dass man als Analytiker nun auch mit arbeitsteilig produzierten und in ihrer Botschaft widersprüchlichen Formen von recipient design konfrontiert werden kann. In Analogie zur multimodalen Struktur von Turn-Taking-Organisation (Schmitt 2005), bei der ein etablierter Sprecher etwa durch verbale Abstinenz anerkannt, gleichzeitig durch gestisch-mimische Verhaltensweisen jedoch in Frage gestellt werden kann, ist das Folgende möglich: Hinsichtlich der multimodalen Repräsentanz eines Gegenüber sind Formen denkbar, bei denen sich die Informationen auf der Ebene einzelner Ausdrucksressourcen widersprechen oder zumindest ,funktional widersprüchlich“" sein können.

Überträgt man die multimodale Perspektive auf die dreiteilige Sequenz der Intersubjektivitätskonstitution (Angebot - Reaktion - Ratifikation), ist die Wahrscheinlichkeit groß, dass man häufig keine reine verbale Dreiteiligkeit vorfindet, sondern Formen, bei denen eine (oder auch mehrere) Position(en) der dreiteiligen Sequenz der Intersubjektivitätskonstitution ausschließlich visuell wahrnehmbar realisiert wird (werden). Diesbezüglich ist mit einer großen Variabilität der Realisierungsformen zu rechnen. Es wird Fälle geben, bei denen vor allem die Ratifikation, das heißt der dritte Zug der Intersubjektivitätskonstituti- 
on, häufig nur visuell wahrnehmbar realisiert wird. Unsere Analyse hat gezeigt, dass auch Angebote zur Evozierung eines gewünschten recipient design im Modus des körperlichen Ausdrucks vollzogen werden können.

Die multimodale Qualität des recipient design sowie die analyseimplikative Egalität aller Ausdrucksressourcen in der multimodalen Konzeption führen dazu, dass recipient design strukturell durch eine formale Diffusität charakterisiert ist. Eine Kopfbewegung, eine Gestikulation oder eine Blickveränderung, deren Relevanz für das recipient design im Zweifelsfalle erst noch geklärt werden muss, besitzen nicht die Klarheit von Wortgrenzen. Die Spezifik des holistischen Vollzuges ist daher eher das bestimmende Merkmal der multimodalen Sicht auf recipient design als die Lokalität und die scharfe Konturiertheit verbaler Elemente.

\subsection{Einheitenkonstitution}

Mit der multimodalen Qualität des recipient design hängt die Frage der Einheitenkonstitution zusammen. Wie und auf der Grundlage welcher Annahmen können wir entscheiden, welcher Ausschnitt aus dem Interaktionsgeschehen der Analyse des recipient design zugrunde gelegt werden soll? Aufgrund der zurückliegenden Ausführungen und der produzierten fallanalytischen Einsichten ist klar, dass die Antwort auf die Frage, welche Verhaltensaspekte und welche interaktionsstrukturellen und aktivitätsspezifischen Aspekte für die Rekonstruktion des recipient design relevant sind, nicht evident und auch nicht oberflächennah, d.h. an leicht identifizierbaren Phänomenen gegeben werden kann.

Die Analyseeinheit muss - da man sich nicht auf Aspekte der Äußerungsstruktur wie etwa Personen- oder Orts- und Raumreferenz stützen kann - in der analytischen Auseinandersetzung mit den Daten und auf der Grundlage analytischer Investition konstituiert werden. Dabei zeigen Erfahrungen bei der Analyse in Schmitt/Deppermann (2009), dass dabei vor allem die Rekonstruktion des relevanten Kontextes eine wesentliche Rolle spielt. Die Frage ist also, wo der für das recipient design relevante Anker im zurückliegenden Interaktionsgeschehen liegt. Kann man eine solche Verankerung nicht finden, verweist dies im Regelfall auf die Relevanz interaktiver Vorgängigkeit und damit auf situationstranszendierende Grundlagen des recipient design. Eine solche kontextuelle Rekonstruktion, die einen von einem auffälligen Phänomen in die zurückliegende Interaktionsgeschichte führt, ist notwendig, da das recipient design im Rahmen einer zumindest dreischrittigen Aushandlung der Intersubjektivitätskonstitution erfolgt. Eine kontextuelle Rekonstruktion ist in vielen Fällen die wesentliche Voraussetzung für die Identifikation des für das recipient design relevanten Bezugspunktes. Das gilt zwingend für alle Fälle, in denen es empirische Evidenz dafür gibt, dass das recipient design ,brought about“ ist, also aus dem Interaktionsverlauf in Reaktion auf interaktives Verhalten emergiert.

Auch die Tatsache, dass das multimodale Beteiligungsdesign in Mehrpersonen-Konstellationen auch bei der Bearbeitung von allgemeinen Anforderungen der Interaktionskonstitution wie etwa der Organisation von Beteiligungsweisen und der gleichzeitigen Adressierung unterschiedlicher Beteiligter relevant werden kann, wirkt sich erschwerend auf die Einheitenkonstitution aus.

Das Problem der Einheitenkonstitution liegt weiterhin darin begründet, dass die multimodale Qualität des relevanten Verhaltens sowohl für das recipient design als auch für das Verhalten, auf das es reagiert, eine schnelle Identifikation erschwert. Dies umso mehr, 
wenn man auch daran interessiert ist, die Genese des recipient design zu rekonstruieren und dabei dessen sukzessive Entwicklung hin zum manifesten Ausdruck, der vielleicht Startpunkt der analytischen Beschäftigung war.

Dass es bei der Einheitenkonstitution offene oder undeutliche Ränder gibt, verdeutlicht auch unser Fall. Hier kann man durchaus Argumente dafür und dagegen formulieren, dass der konstante Blick des Lehrers zur Tür zu Beginn des Ausschnitts, den er mimisch starr realisiert, bereits zum recipient design gehört oder nicht.

\subsection{Benennung}

Aufgrund der komplexen multimodalen Struktur und der strukturellen Reflexivität des multimodalen Konzeptes muss geklärt werden, ob bei der Übernahme und Adaption konzeptkonstitutiver Bestandteile die Benennung und die damit verbundene analytische Transparenz beibehalten werden kann oder ob man das Konzept nicht konsequenterweise umbenennen muss. Inwieweit diese Notwendigkeit besteht und welche Begriffe dabei erfolgversprechende Kandidaten sind, hängt auch vom jeweiligen Fokus ab, den man abhängig vom spezifischen Erkenntnisinteresse - hinsichtlich der Repräsentanz des Gegenübers definieren wird. Grundsätzlich sollte man sich auf der Suche nach einer phänomenadäquaten Begrifflichkeit daran orientieren, den jeweiligen Erkenntnisfokus in der Begriffsbildung selbst analytisch transparent zu machen.

Diesbezüglich wäre beispielsweise im Zusammenhang mit einem Erkenntnisfokus „Positionierungsimplikationen“ die bereits diskutierte Möglichkeit (7.3) zu prüfen, generisch von einem participant design zu sprechen und dieses durch den Bezug definierenden Zusatz fremdbezogen oder selbstbezogen zu qualifizieren.

Ist man hingegen primär an der interaktiven Verdeutlichung interessiert, mit der Beteiligte ausweisen, an wen sie konkrete Äußerungen richten oder für sie etablierte konditionelle Relevanzen bearbeiten oder wen sie selbst zu spezifischen Reaktionen verpflichten wollen, etabliert man einen anderen Fokus. In solchen Fällen ist es sinnvoll, etwa auf „Adressatenzuschnitt" als deutschsprachige Alternative zu recipient design zurückgreifen, wie das Deppermann/Blühdorn (2013) vorschlagen.

Ist man in einem generelleren Sinne an ,Verfahren der Repräsentanz relevanter Interaktionsbeteiligter“ interessiert, suggerieren die Begriffsbestandteile „design“ und „Zuschnitt“ ein - aufgrund ihrer monomodalen Verweisqualität durchaus plausibles - hohes Ausmaß an Bewusstheit und Kontrolle des Designers über seine Verhaltensspezifik zu einem bestimmten Zeitpunkt. Bei der in unserer Fallanalyse nachgewiesenen komplexen, multiaspektuellen und zeitlich asynchronen Ressourcenlage ist es wichtig, eine begriffliche Transparenz anzusteuern, welche keine Annahmen diesbezüglich macht bzw. nicht in diese Richtung suggestiv ist. Da wir für den Moment über einen solchen Terminus noch nicht verfügen, behelfen wir uns - auch um die strukturelle Affinität mit der konversationsanalytischen Vorstellung zum Ausdruck zu bringen - mit dem Arbeitskonzept ,multimodales recipient design“. 
Recipient design im hier präsentierten Verständnis als Repräsentation von relevanten Interaktionsbeteiligten im eigenen interaktiven Verhalten sollte unseres Erachtens systematisch im Zusammenhang mit übergeordneten Theoriezusammenhängen reflektiert werden, um dessen interaktionstheoretischen Status klarer zu konturieren.

Die zurückliegenden Ausführungen haben bereits Hinweise darauf produziert, welche Theoriezusammenhänge hierfür relevant sein können:

Es geht einerseits um die Vorstellung von Verstehensdokumentationen als empirische Evidenz der für das Zustandekommen von Interaktion konstitutiven Herstellung einer tragfähigen Intersubjektivität. Andererseits ist die online-analytische Vorstellung wichtig, nach der in grundsätzlicher und konstitutiver Weise das eigene interaktive Verhalten immer unter kontinuierlicher, interpretativer Berücksichtigung des vorgängigen und gleichzeitigen Verhaltens relevanter anderer Interaktionsbeteiligter realisiert wird (Goodwin 1980; Dausendschön-Gay/Krafft 2000; Mondada 2006).

In einem solchen doppelten Theoriebezug wird es möglich, Affinitäten und Gemeinsamkeiten der verstehensdokumentarischen und online-analytischen Perspektive für eine multimodale Konzeption von recipient design produktiv zu machen.

Die Relevanz beider Perspektiven wurde implizit bereits in den zurückliegenden Ausführungen bei der dreischrittigen Intersubjektivitätskonstitution und bei der Frage nach dem Verhältnis von „brought along“ und „brought about“ deutlich. Es ist nun eigentlich nur noch ein kleiner Schritt - ausgehend von der hier präsentierten allgemeinen Bestimmung von „recipient design als multimodale Repräsentanz des Gegenübers im eigenen Verhalten" sowohl mit den online-analytischen Grundlagen dieser Repräsentanz und deren verstehensdokumentarischen Qualität das Nachdenken über recipient design explizit an diese Vorstellungen anzuschließen.

In diesem theoretischen An- und Zusammenschluss kann man auf der Grundlage der bei unserer Fallanalyse produzierten Einsichten recipient design wie folgt neu bestimmen:

Mit recipient design konzeptualisieren wir

- eine online-analytisch konstituierte Verstehensdokumentation,

- in der interaktiv und/oder sozial relevante Eigenschaften von Interaktionsteilnehmern aus der Sicht („Orientierung“ und „Sensitivität“ nach Sacks/Schegloff/Jefferson 1974) eines Interaktionsbeteiligten

- entweder für das konkrete interaktive Gegenüber oder - in Mehrparteien-Konstellationen - für andere Anwesende

- in intersubjektiv relevanter Weise zum Ausdruck gebracht und als

- relevante Orientierungshilfen für die adäquate Einschätzung des Produzenten des recipient design angeboten werden.

Recipient design würde damit als empirisch zu untersuchende, theoretisch gehaltvolle Manifestation der für Interaktionskonstitution zentralen Herstellung und Gewährleistung von Intersubjektivität in den Fokus rücken. Eine solche theoretische Neu-Kontextualisierung würde nicht nur recipient design als eigenständigen Untersuchungsgegenstand aufwerten. Sie würde auch Einblicke in die tatsächliche Bandbreite und das Varianzspektrum wahr- 
nehmbarer Verstehensdokumentationen und in die Permanenz online-analytischer „Arbeit““ als Teil interaktiver Beteiligung produzieren: eine klassische Win-win-Situation also.

\subsection{Handlungsfunktionalität/Rhetorische Qualität}

Ein wichtiger, aber noch weitgehend unreflektierter Punkt ist die Frage nach den rhetorischen Implikationen des recipient design. Wird recipient design von Beteiligten im Rahmen gesprächsrhetorischer Orientierungen eingesetzt? Wird also das Gegenüber in einer für die Verfolgung eigener Handlungsrelevanzen funktionalen Weise positioniert und für andere Beteiligte angeboten? Kann man diese Frage empirisch fundiert mit ja beantworten, muss man die Möglichkeit konzeptuell integrieren, dass recipient design dann in einem sehr weitgehenden Sinne eine ausschließliche Konstruktion des Designers ist, die sich gänzlich von der tatsächlichen interaktiven und sozialen Ausstattung des Gegenüber abgelöst hat.

Recipient design wäre dann nicht nur ein handlungsfaktisch kaum zu hintergehender Bestandteil zielorientierten Handelns, sondern könnte eine für die Zielorientierung wesentliche Voraussetzung darstellen. Ein spezifisches recipient design, das das Gegenüber mit interaktiven, sozialen und kulturellen Aspekten ausstattet, kann dazu benutzt werden, Handlungsmöglichkeiten des Gegenübers einzuschränken, seine Glaubwürdigkeit und seinen egalitären Beteiligungsstatus in Frage zu stellen, wodurch es interaktiv ins Hintertreffen geraten kann.

\section{Literatur}

Auer, Peter (1992). Introduction: John Gumperz' approach to contextualization. In: Auer, Peter/di Luzio, Aldo (Hg.): The contextualization of language. Amsterdam/Philadelphia: Benjamins. S. 1-37.

Bell, Allan (2001): Back in style: reworking audience design. In: Eckert, Penelope/Rickford, John R. (Hg.): Style and sociolinguistic variation. Cambridge: Cambridge University Press. S. 139-169.

Birdwhistell, Ray, L. (1970): Kinesics and context: essays on body-motion communication. Philadelphia: University of Pennsylvania Press.

Birdwhistell, Ray, L. (1984): Kinesik. In: Scherer, Klaus R./Wallbot, Harald G. (Hg.): Nonverbale Kommunikation: Forschungsberichte zum Interaktionsverhalten. 2. Aufl. Weinheim: Beltz. S. 192-202.

Brown, Penelope/Levinson, Stephen C. (1987): Politeness: some universals in language usage. Cambridge: Cambridge University Press.

Cicourel, Aaron V. (1975): Sprache in der sozialen Interaktion. München: List.

Clark, Herbert H. (1992): Arenas of language use. Chicago: Chicago University Press.

Clark, Herbert H. (1996a): Using language. Cambridge: Cambridge University Press.

Clark, Herbert H. (1996b): Communities, commonalities and communication. In: Gumperz, John J./Levinson, Stephen C. (Hg.): Rethinking linguistic relativity. Cambridge: Cambridge University Press. S. 324-355.

Coupland, Nicolas (2007): Style: language variation and identity. Cambridge: Cambridge University Press.

Dausendschön-Gay, Ulrich/Krafft, Ulrich (2000): On-line-Hilfen für den Hörer: Verfahren zur Orientierung der Interpretationstätigkeit. In: Wehr, Barbara/Thomaßen, Helga (Hg.): Diskursanalyse. Untersuchungen zum gesprochenen Französisch. Frankfurt/Main u.a.: Lang. S. 17-55.

Deppermann, Arnulf (2008): Verstehen im Gespräch. In: Eichinger, Ludwig/Kämper, Heidrun (Hg.): Sprache - Kognition - Kultur. Berlin: de Gruyter. S. 225-261. (= Jahrbuch des Instituts für Deutsche Sprache 2007).

Deppermann, Arnulf (2009): Verstehensdefizit als Antwortverpflichtung: Interaktionale Eigenschaften der Modalpartikel denn in Fragen. In: Günthner, Susanne/Bücker, Jörg (Hg.): Grammatik im Gespräch. Konstruktionen der Selbst- und Fremdpositionierung. Berlin/New York: de Gruyter. S. 23-56.

Deppermann, Arnulf (erscheint): How to get a grip on identities-in-interaction: (What) Does ,positioning ${ }^{*}$ offer more than 'membership categorization"? Evidence from a mock story: In: Narrative Inquiry 23 (2). 
Deppermann, Arnulf/Blühdorn, Hardarik (2013): Negation als Verfahren des Adressatenzuschnitts: Verstehenssteuerung durch Interpretationsrestriktionen. In: Deutsche Sprache 41, S. 6-30.

Deppermann, Arnulf/Schmitt, Reinhold (2009): Verstehensdokumentationen: Zur Phänomenologie von Verstehen in der Interaktion. In: Deutsche Sprache 36, S. 220-245.

Fetzer, Anita/Fischer, Kerstin (Hg.) (2007): Lexical markers of common ground. Amsterdam: Elsevier.

Garfinkel, Harold (1967): Studies in ethnomethodology. Englewood Cliffs, NJ: Prentice-Hall.

Giddens, Anthony (1976): New rules of sociological method. London: Hutchinson.

Giles, Howard/Coupland, Justine/Coupland, Nicolas (Hg.) (1991): Contexts of accommodation. Cambridge: Cambridge University Press.

Goodwin, Charles (2003): Pointing as situated practice. In: Kita, Sotaro (Hg.): Pointing: where language, culture and cognition meet. Mahwah, NJ: Erlbaum. S. 217-241.

Goodwin, Marjorie H. (1980): Processes of mutual monitoring implicated in the production of description sequences. In: Sociological Inquiry 50, S. 303-317.

Gumperz, John J. (2002): Sharing common ground. In: Keim, Inken/Schütte, Wilfried (Hg.): Soziale Welten und kommunikative Stil: Festschrift für Werner Kallmeyer zum 60. Geburtstag. Tübingen: Narr. S. 47-56. (= Studien zur Deutschen Sprache 22).

Hausendorf, Heiko (2003): Deixis and speech situation revisited. The mechanism of perceived perception. In: Lenz, Friedrich (Hg.): Deictic conceptualisation of space, time and person. Amsterdam/Philadelphia: Benjamins. S. 249-269.

Hausendorf, Heiko/Mondada, Lorenza/Schmitt, Reinhold (Hg.) (2012): Raum als interaktive Ressource. Tübingen: Narr. (= Studien zur Deutschen Sprache 62).

Hausendorf, Heiko/Schmitt, Reinhold (2013): Interaktionsstruktur und Sozialtopografie. Umrisse einer raumlinguistischen Programmatik. Arbeitspapiere des UFSP Sprache und Raum (SpuR) Nr. 01. Zürich. Internet: www.spur.uzh.ch/research.html.

Heidtmann, Daniela (2009): Multimodalität der Kooperation im Lehr-Lern-Diskurs: Wie Ideen für Filme entstehen. Tübingen: Narr. (= Studien zur Deutschen Sprache 50).

Heidtmann, Daniela/Föh, Marie-Joan (2007): Verbale Abstinenz als Form interaktiver Beteiligungsweise. In: Schmitt, Reinhold (Hg.), S. 263-292.

Heidtmann, Daniela/Schmitt, Reinhold (2010): Synchronität als Kooperationsmodus im pädagogischen Diskurs: Das Beispiel Kinderkonferenz. In: Kallmeyer, Werner/Reuter, Ewald/Schopp, Jürgen (Hg.): Perspektiven auf Kommunikation. Berlin: Saxa. S. 27-68.

Heidtmann, Daniela/Schmitt, Reinhold (2011): Interaktives Diktieren. Komplexe Anforderungen im Epochen-Unterricht. In: Schmitt, Reinhold (Hg.), S. 179-224.

Heilman, Samuel C. (1979): Communication and interaction: a parallel in the theoretical outlooks of Erving Goffman and Ray Birdwhistell. In: Communication 4, S. 221-234.

Heritage, John (2007): Intersubjectivity and progressivity in person (and place) reference. In: Enfield, Nick J./Stivers, Tanya (Hg.): Person reference in interaction. Cambridge: Cambridge University Press. S. 255-280.

Hinnenkamp, Volker (1989): Interaktionale Soziolinguistik und interkulturelle Kommunikation: Gesprächsmanagement zwischen Deutschen und Türken. Tübingen: Niemeyer.

Holly, Werner (1990): Politikersprache. Tübingen: Niemeyer.

Kallmeyer, Werner (1996): Einleitung. Was ist Gesprächsrhetorik? In: Ders. (Hg.): Gesprächsrhetorik. Rhetorische Verfahren im Gesprächsprozeß. Tübingen: Narr. S. 7-18. (= Studien zur deutschen Sprache 4).

Kallmeyer, Werner/Schmitt, Reinhold (1996): Forcieren oder: Die verschärfte Gangart. Zur Analyse von Kooperationsformen im Gespräch. In: Kallmeyer, Werner (Hg.): Gesprächsrhetorik. Rhetorische Verfahren im Gesprächsprozeß. Tübingen: Narr. S. 20-118. (= Studien zur deutschen Sprache 4).

Kendon, Adam (1990a): Conducting interaction: patterns of behavior in focused encounters. Cambridge: Cambridge University Press. 
Kendon, Adam (1990b): Some context for context analysis: a view of the origins of structural studies of face-to-face interaction. In: Kendon, Adam (1990a), S. 15-49.

Kühn, Peter (1995): Mehrfachadressierung. Tübingen: Niemeyer.

Lerner, Gene (2003): Selecting next speaker: the context sensitive operation of a context-free organization. Language in Society 32 (2), S. 177-201.

Lucius-Hoene, Gabriele/Deppermann, Arnulf (2002): Rekonstruktion narrativer Identität: ein Arbeitsbuch zur Analyse narrativer Interviews. Opladen: Verlag für Sozialwissenschaften.

Lucius-Hoene, Gabriele/Deppermann, Arnulf (2004): Narrative Identität und Positionierung. In: Gesprächsforschung 5, S. 166-183. Internet: www.gespraechsforschung-ozs.de/heft2004/ga-lucius.pdf.

Malone, Martin J. (1997): Worlds of talk: the presentation of self in everyday conversation. Cambridge: Polity Press.

Mondada, Lorenza (2006): Particapants' online analysis and multimodal practices: projecting the end of the turn and the closing of the sequence. In: Discourse Studies 8 (1), S. 117-129.

Mondada, Lorenza (2007): Multimodal resources for turn-taking: pointing and the emergence of possible next speakers. In: Discourse Studies 9 (2), S. 195-226.

Mondada, Lorenza/Schmitt, Reinhold (Hg.) (2010): Situationseröffnungen: Zur multimodalen Herstellung fokussierter Interaktion. Tübingen: Narr. (= Studien zur Deutschen Sprache 47).

Müller, Cornelia/Bohle, Ulrike (2007): Das Fundament fokussierter Interaktion. Zur Vorbereitung und Herstellung von Interaktionsräumen durch körperliche Koordination. In: Schmitt, Reinhold (Hg.), S. 129-165.

Pomerantz, Anita (1980): Telling my side: "Limited Access" as a "Fishing" device. In: Sociological Inquiry 50 (3-4), S. 186-198.

Pustički, Nikolina/Schmitt, Reinhold (i.V.): Zur Ko-Konstruktion einer amüsanten Unterbrechung während einer argumentativen Auseinandersetzung. Erscheint in: Dausendschön-Gay, Ulrich/Gülich, Elisabeth/ Krafft, Ulrich (Hg.): Ko-Konstruktionen als interaktive Verfahren.

Sacks, Harvey (1992): Lectures on conversation. 2 Bde. Oxford: Blackwell.

Sacks, Harvey/Schegloff, Emanuel A. (1979): Two preferences in the organization of reference to persons in conversation and their interaction. In: Psathas, George (Hg.): Everyday language: studies in ethnomethodology. New York: Irvington. S. 15-21.

Sacks, Harvey/Schegloff, Emanuel A./Jefferson, Gail (1974): A simplest systematics for the organization of turn-taking in conversation. In: Language 50 (4), S. 696-735.

Scheflen, Albert E. (1964): The significance of posture in communication systems. In: Psychiatry 27, S. 316-313.

Scheflen, Albert E. (1972): Body language and social order: communication as behavioral control. Englewood Cliffs, NJ: Prentice-Hall.

Schegloff, Emanuel (1972): Notes on a conversational practice: formulating place. In: Sudnow, David (Hg.): Studies in social interaction. New York: Free Press. S. 75-119.

Schegloff, Emanuel (2007): Conveying who you are: the presentation of self, strictly speaking. In: Enfield, Nick J./Stivers, Tanya (Hg.): Person reference in interaction: linguistic, cultural and social perspectives. Cambridge: Cambridge University Press. S. 123-148.

Schmitt, Reinhold (1992a): Die Schwellensteher. Sprachliche Präsenz und sozialer Austausch in einem Kiosk. Tübingen: Narr.

Schmitt, Reinhold (1992b): Das Konzept der Präsenzfigur. Ein Beitrag zur Integration von Konversationsanalyse und objektiver Hermeneutik. In: Protosoziologie 3, S. 123-131, S. 141-143.

Schmitt, Reinhold (2001): Von der Videoaufzeichnung zum Konzept „Interaktives Führungshandeln“: Methodische Probleme einer inhaltlich orientierten Gesprächsanalyse. In: Gesprächsforschung 2, S. 141-192 (www.gespraechsforschung-ozs.de).

Schmitt, Reinhold (2004): Die Gesprächspause: „Verbale Auszeiten“ aus multimodaler Perspektive. In: Deutsche Sprache 32, S. 56-84. 
Schmitt, Reinhold (2005): Zur multimodalen Struktur von turn-taking. In: Gesprächsforschung 6, S. 17-61 (www.gespraechsforschung-ozs.de).

Schmitt, Reinhold (Hg.) (2007): Koordination. Analysen zur multimodalen Interaktion. Tübingen: Narr. (= Studien zur Deutschen Sprache 38).

Schmitt, Reinhold (2007a): Von der Konversationsanalyse zur Analyse multimodaler Interaktion. In: Kämper, Heidrun/Eichinger, Ludwig M. (Hg.): Sprach-Perspektiven. Germanistische Linguistik und das Institut für Deutsche Sprache. Tübingen: Narr. S. 395-417. (= Studien zur Deutschen Sprache 40).

Schmitt, Reinhold (2007b): Theoretische und methodische Implikationen der Analyse multimodaler Interaktion. In: Holly, Werner/Paul, Ingwer (Hg.): Medialität und Sprache. Bielefeld: Aisthesis. S. 26-53. (= Mitteilungen des Deutschen Germanistenverbandes 54 (1)).

Schmitt, Reinhold (2008): Die Schwellensteher. Sprachliche Präsenz und interaktiver Austausch in einem Kiosk. Verlag für Gesprächsforschung (Neuauflage): http://www.verlag-gespraechsforschung.de/2008/ schmitt2.htm.

Schmitt, Reinhold (2009): Schülerseitiges Interaktionsmanagement: Initiativen zwischen supportiver Strukturreproduktion und Subversion. In: Gesprächsforschung 10, S. 20-69. www.gespraechsforschung-ozs. de/heft2009/ga-schmitt.pdf.

Schmitt, Reinhold (2010): Verfahren der Verstehensdokumentation am Filmset: Antizipatorische Initiativen und probeweise Konzeptrealisierung. In: Deppermann, Arnulf et al. (Hg.): Verstehen in professionellen Handlungsfeldern. Tübingen: Narr. S. 210-362. (= Studien zur Deutschen Sprache 52).

Schmitt, Reinhold (Hg.) (2011): Unterricht ist Interaktion! Analysen zur De-facto-Didaktik. Mannheim: amades.

Schmitt, Reinhold (2012a): Gehen als situierte Praktik: „Gemeinsam gehen“ und „hinter jemandem herlaufen“. Erscheint in: Gesprächsforschung 13, S. 1-44 (www.gespraechsforschung-ozs.de).

Schmitt, Reinhold (2012b): Zur Multimodalität von Unterstützungsinteraktion. In: Ders. (Hg.): Empirische Perspektiven auf Interaktion. Themenheft Deutsche Sprache 40, S. 343-371.

Schmitt, Reinhold (2012c): Körperlich-räumliche Grundlagen interaktiver Beteiligung: Das Konzept „Interaktionsensemble“. In: Hausendorf, Heiko/Mondada, Lorenza/Schmitt, Reinhold (Hg.), S. 37-87.

Schmitt, Reinhold (2012d): Zur Multimodalität von Unterstützungsinteraktion In: Schmitt (Hg.): Analytische Perspektiven auf Interaktion. Themenheft Deutsche Sprache 40, S. 343-371.

Schmitt, Reinhold (2013): Körperlich-räumliche Grundlagen der Interaktion. Tübingen: Narr. (= Studien zur Deutschen Sprache 64).

Schmitt, Reinhold (erscheint): Zum „Be-Greifen“ relevanter Aspekte der räumlichen Umgebung. In: Festschrift anlässlich des 30-jährigen Bestehens des Instituts für Philologie und interkulturelle Kommunikation der Universität Wolgograd. Wolgograd.

Schmitt, Reinhold/Deppermann, Arnulf (2007): Monitoring und Koordination als Voraussetzungen der multimodalen Konstitution von Interaktionsräumen. In: Schmitt, Reinhold (Hg.), S. 95-128.

Schmitt, Reinhold/Deppermann, Arnulf (2009): "damit sie mich verstehen”: Genese, Verfahren und recipient design einer narrativen Performance. In: Buss, Mareike et al. (Hg.): Theatralität des sprachlichen Handelns. München: Fink. S. 83-112.

Schmitt, Reinhold/Deppermann, Arnulf (2010): Die multimodale Konstitution eines imaginären Raums als interaktive Problemlösung. In: Deppermann, Arnulf/Linke, Angelika (Hg.): Sprache intermedial. Berlin/ New York: de Gruyter. S. 199-241. (= Jahrbuch des Institut für Deutsche Sprache 2009).

Selting, Margret et al. (2009): Gesprächsanalytisches Transkriptionssystem 2 (GAT 2). In: Gesprächsforschung 10, S. 353-402 (www.gespraechsforschung-ozs.de).

Dr. Reinhold Schmitt

Institut für Deutsche Sprache

Postfach 101621

D-68016 Mannheim

reinhold.schmitt@ids-mannheim.de
Dr. Ralf Knöbl

Institut für Deutsche Sprache

Postfach 101621

D-68016 Mannheim

knoebl@ids-mannheim.de 


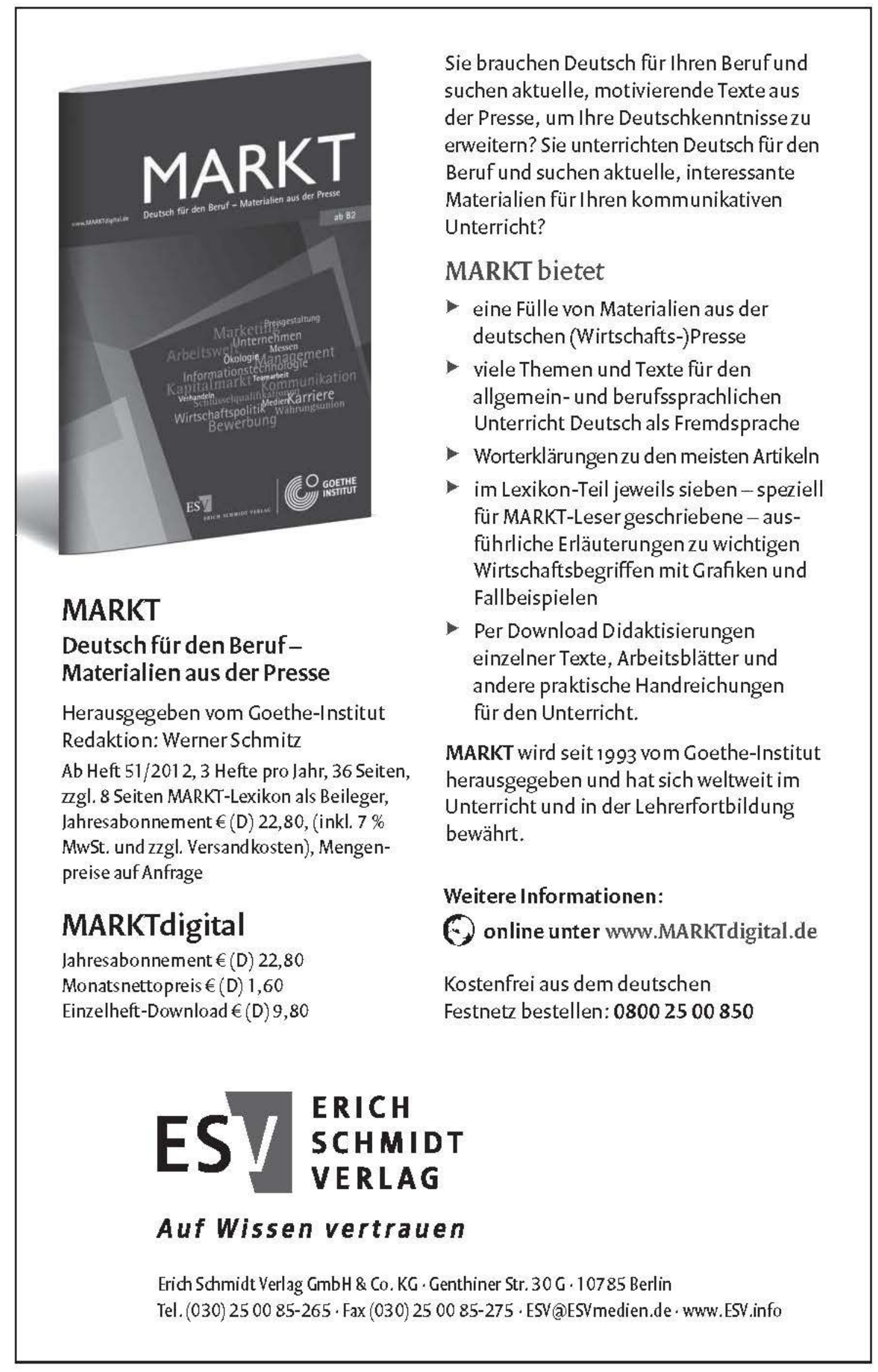

